\title{
Label-Free Quantitative Proteomics and Substrate-Based Mass Spectrometry Imaging of Xenobiotic Metabolizing Enzymes in Ex Vivo Human Skin and a Human Living Skin Equivalent Model
}

\author{
(D) Narciso Couto, Jillian R.A. Newton, Cristina Russo, @Esther Karunakaran, (1)Brahim Achour, \\ (1)Zubida M. Al-Majdoub, James Sidaway, Amin Rostami-Hodjegan, Malcolm R. Clench, \\ and (1) Jill Barber
}

Department of Chemical and Biological Engineering (N.C., E.K.) and Sheffield Collaboratorium for Antimicrobial Resistance and Biofilms (SCARAB) (N.C., E.K.), University of Sheffield, Sheffield, United Kingdom; Centre for Applied Pharmacokinetic Research,

University of Manchester, Manchester, United Kingdom (N.C., B.A., Z.M.A.-M., A.R.-H., J.B.); Centre for Mass Spectrometry Imaging, Biomolecular Sciences Research Centre, Sheffield Hallam University, Sheffield, United Kingdom (J.R.A.N., C.R., M.R.C.); Phenotox Ltd., Bollington, United Kingdom (J.S.); and Certara UK Limited (Simcyp Division), Sheffield, United Kingdom (A.R.-H.)

Received July 3, 2020; accepted October 8, 2020

\begin{abstract}
We report for the first time label-free quantification of xenobiotic metabolizing enzymes (XME), transporters, redox enzymes, proteases, and nucleases in six human skin explants and a threedimensional living skin equivalent model from LabSkin. We aimed to evaluate the suitability of LabSkin as an alternative to animal testing for the development of topical formulations. More than 2000 proteins were identified and quantified from total cellular protein. Alcohol dehydrogenase $1 \mathrm{C}$, the most abundant phase I XME in human skin, and glutathione S-transferase pi 1, the most abundant phase II XME in human skin, were present in similar abundance in LabSkin. Several esterases were quantified and esterase activity was confirmed in LabSkin using substrate-based mass spectrometry imaging. No cytochrome P450 (P450) activity was observed for the substrates tested, in agreement with the proteomics data, where the cognate P450s were absent in both human skin and LabSkin. Label-free protein quantification allowed insights into other related processes such as redox homeostasis and proteolysis. For example,
\end{abstract}

Introduction

In Europe, testing cosmetic products on animals has been banned since 2009 because of the seventh amendment of the European Union Cosmetics Directive. Animals are, however, still used for testing the majority of new topical drugs. Efforts have been made worldwide to

This work was funded by The National Centre for the Replacement, Refinement and Reduction of Animals in Research (NC3Rs) through the Metaboderm CRACK IT challenge. This work was also supported by Croda Ltd. The authors thank the ChELSI Institute, University of Sheffield, for access to liquid chromatography-tandem mass spectrometry instrumentation (funded under BBSRC Grant: BB/M012166/1; and EPSRC Grant: EP/E036252/1).

https://doi.org/10.1124/dmd.120.000168. the most abundant antioxidant enzymes were thioredoxin and peroxiredoxin-1. This systematic determination of functional equivalence between human skin and LabSkin is a key step toward the construction of a representative human in vitro skin model, which can be used as an alternative to current animal-based tests for chemical safety and for predicting dosage of topically administered drugs.

\section{SIGNIFICANCE STATEMENT}

The use of label-free quantitative mass spectrometry to elucidate the abundance of xenobiotic metabolizing enzymes, transporters, redox enzymes, proteases, and nucleases in human skin enhance our understanding of the skin physiology and biotransformation of topical drugs and cosmetics. This will help to develop mathematical models to predict drug metabolism in human skin and to develop more robust in vitro engineered human skin tissue as alternatives to animal testing.

ABBREVIATIONS: ABC, ATP-binding cassette; ADH, alcohol dehydrogenase; AKR, aldo-ketoreductase; ALDH, aldehyde dehydrogenase; AOC, amine oxidase; BLVRA, biliverdin reductase A; CBR, carbonyl reductase; CES1, carboxylesterase 1; COMT, catechol-O-methyltransferase; CST, cystatin; CTS, cathepsin; CV, coefficient of variation; 3D, three dimensional; EPHX, epoxide hydrolase; FDR, false discovery rate; GST, glutathione S-transferase; HPA, Human Protein Atlas; MALDI, matrix-assisted laser desorption ionization; MSI, mass spectrometry imaging; P450, cytochrome P450; PRDX, peroxiredoxin; SLC, solute carrier; SOD, superoxide dismutase; SULT, sulfotransferase; TXN, thioredoxin; UGT, UDPglucuronosyltransferase; XME, xenobiotic metabolizing enzyme. 
analyze using traditional proteomic techniques owing to the high lipid content, and the insolubility and extensive crosslinking of proteins. This can complicate the isolation and digestion of proteins for analysis using mass spectrometry techniques. Consequently, the roles of XME and drug transporters in the human skin remain to be elucidated. We therefore set out to measure the levels of phase I and II XMEs and phase III drug transporters in in vitro skin models and compare them to human skin.

During xenobiotic metabolism, phase I and phase II enzymes activate xenobiotics by adding or revealing polar groups. Phase I enzymes include cytochrome P450 (P450) enzymes, aldehyde oxidases, aldehyde dehydrogenases (ALDHs), aldo-ketoreductases (AKRs), alcohol dehydrogenases (ADHs), esterases, flavin-containing monooxygenases, and cyclooxygenases. Enzymes from phase II include glutathione S-transferases (GSTs), UDP-glucuronosyltransferases (UGTs), sulfotransferases (SULTs), N-acetyltransferases, and methyltransferases. The activated xenobiotics are subsequently transported to the bloodstream by a variety of phase III transporters from the ATP-binding cassette (ABC) and solute carrier (SLC) families (Kazem et al., 2019).

Although these enzymes and transporters are the main players in xenobiotic disposition, biotransformation and transportation of xenobiotics are complex and dynamic processes where other enzymes and proteins can also play a direct or indirect role. For example, phase I metabolism produces reactive oxygen species, which, without control, cause oxidative stress and impair protein function (Couto et al., 2013, 2016). However, the mechanism of reactive oxygen species generation during xenobiotic metabolism, association of XME in diseases, and the role of antioxidants and antioxidant enzymes in determining the effectiveness of xenobiotic metabolism have not been fully established. Given the important role of the antioxidant system in mediating the pharmacodynamics of drugs, it is necessary to understand antioxidant response to xenobiotics, the role of reactive oxygen species in toxicity, and the role of antioxidant enzymes in modulating xenobiotic metabolism. It is also important to understand the roles of proteases, peptidases, and protease inhibitors in skin because both cosmetics and emerging topical drug therapies increasingly incorporate bioactive peptides. In the human skin, the activity of peptidases and their inhibitors is tightly regulated. A breakdown in these regulatory mechanisms has been associated with a broad range of conditions such as cancer (Hu et al., 2007) and inflammation (Overall and Dean, 2006).

Mass spectrometry-based quantitative proteomics is considered an excellent strategy to investigate metabolism in biologic systems by quantifying protein abundance (Parker et al., 2014; Russo et al., 2016; Flores et al., 2019; Raut et al., 2019). To evaluate the potential for using in vitro laboratory models of human skin as viable alternatives to animal testing, a mass spectrometry-based approach using both label-free quantitative proteomics and matrix-assisted laser desorption ionization (MALDI) imaging (Russo et al., 2018) was undertaken in this study. The abundance of enzymes involved in xenobiotic metabolism including antioxidant enzymes, proteases, peptidases, and nucleases were determined in an in vitro 3D human skin model (hereafter referred to as LabSkin) and compared with six human skin sections.

\section{Materials and Methods}

\section{Chemicals}

Unless, otherwise indicated, all chemicals were supplied by Sigma-Aldrich (Poole, Dorset, UK) with the highest purity available. Sequencing grade modified trypsin was supplied by Promega (Southampton, UK). All solvents were high-performance liquid chromatography grade and supplied by ThermoFisher Scientific (Paisley, UK).

\section{Human Skin Samples}

Human skin tissues from one male and five female donors, were taken from "healthy" individuals undergoing routine abdominoplasty surgery. These human skin tissues were sourced by the Teaching Hospital from The University of Bradford. Ethics were approved for this study (36-DRMBPY-06-001) by the Independent Ethics Committee. All participants provided written informed consent and sample collection was conducted according to the principles expressed in the Declaration of Helsinki protocols.

\section{D LabSkin Samples}

LabSkin was supplied by LabSkin UK Ltd (York, UK). LabSkin was delivered after 14 days of development in transport culture medium. At the time of delivery, LabSkin was transferred into new deep 12-well plates, suspended in fresh LabSkin maintenance medium, and left to incubate for 24 hours at $37^{\circ} \mathrm{C}$ with $5 \% \mathrm{CO}_{2}$, to normalize the metabolism.

\section{Label-Free Quantification of Proteins}

Sample Preparation. All procedures were performed with sterile equipment and solvents using aseptic techniques. Approximately $3 \mathrm{~g}$ of human skin tissue and $1.5 \mathrm{~g}$ of LabSkin were washed twice with $10 \mathrm{ml}$ of washing buffer $(250 \mathrm{mM}$ phosphate buffer $\mathrm{pH}$ 7.4, $150 \mathrm{mM}$ potassium chloride, $1 \mathrm{mM}$ EDTA). From the human tissues, the subcutaneous layer was removed before skin samples were cut into small pieces with sterile forceps and surgical scalpels. LabSkin was also cut into small pieces. The pieces from the human skin and LabSkin were washed three times with washing buffer and centrifuged at $50 \mathrm{~g}$ for 10 minutes. Samples were incubated overnight at $4{ }^{\circ} \mathrm{C}$ with $10 \mathrm{ml}$ lysis buffer $(25 \mathrm{mM}$ Tris $\mathrm{pH} 7.6,150 \mathrm{mM}$ sodium chloride, $1 \%$ NP40, $0.5 \%$ sodium deoxycholate, $0.1 \%$ SDS, $1 \mathrm{mM}$ EDTA) incorporating enzymatic inhibitor (Roche, UK). Samples were homogenized using a mechanical device (Ultra Turrax T25) using 10 cycles of 30 seconds interspersed with 30 seconds resting time on ice between cycles. After this procedure, the homogenate was centrifuged at $9000 \mathrm{~g}$ for 30 minutes at $4^{\circ} \mathrm{C}$, and the supernatant was collected in a clean tube and clarified using DNAse. Therefore, the protein samples extracted using this strategy are representative of total cellular protein.

Protein Digestion. Protein content in the LabSkin and human skin samples was estimated by a spectrophotometric protein assay using Bradford reagent (ThermoFisher Scientific, Hemel Hempstead, UK) (Bradford, 1976). Analysis was made in triplicate according to the manufacturer's protocol using bovine serum albumin as a standard. Based on the results of the Bradford assay, $100 \mu \mathrm{g}$ of each fraction was spiked with an internal standard protein mixture containing 17 pmol equine myoglobin, 12 pmol bovine cytochrome $c$, and 3 pmol bovine serum albumin. These nonhuman proteins were selected because their low similarity with their human counterparts minimizes interference. To each fraction containing the standards, sodium deoxycholate was added to achieve a final concentration of $10 \%(\mathrm{w} / \mathrm{v})$. The mixture was mixed well and incubated at room temperature for 10 minutes. The filter-aided sample preparation method was used as previously described (Al Feteisi et al., 2018; Couto et al., 2019). Disulfide bridges were reduced by adding $100 \mathrm{mM}$ (final concentration) 1,4-dithiothreitol, and thiols were alkylated by adding $50 \mathrm{mM}$ (final concentration) iodoacetamide. After alkylation and three washes with $1 \mathrm{M}$ urea in $50 \mathrm{mM}$ ammonium bicarbonate $(\mathrm{pH}$ 8.5), protein digestion was performed by adding trypsin (trypsin:protein ratio 1:25 $\mathrm{w} / \mathrm{w})$ followed by overnight incubation at $37^{\circ} \mathrm{C}$. Peptides were recovered by centrifugation $(14,000 \mathrm{~g}, 20$ minutes) first by elution using $100 \mathrm{mM}$ ammonium bicarbonate ( $\mathrm{pH} 8.5$ ) followed by a second elution using $0.5 \mathrm{M}$ sodium chloride. A desalt process was performed using a C18 column (Nest group) as previously described (Couto et al., 2019). The peptides were dried using a vacuum concentrator and stored at $-20^{\circ} \mathrm{C}$ until mass spectrometric analysis.

Liquid Chromatography and Mass Spectrometry. Liquid chromatography-mass spectrometry acquisition was performed using an UltiMate 3000 rapid separation liquid chromatograph (Dionex, Surrey, UK) coupled to an online Q Exactive HF Hybrid Quadrupole-Orbitrap Mass Spectrometer (ThermoFisher Scientific, Bremen, Germany) as previously reported (Couto et al., 2019).

Data Analysis for Label-Free Quantification of Proteins. Protein/peptide identification was performed using MaxQuant version 1.6.2.6. Proteins were identified by searching against a reference human proteome database containing 71,599 entries (UniProt, May 2017). Using MaxQuant, the precursor mass 
tolerance was set to $5 \mathrm{ppm}$, fragment mass tolerance was set to $0.02 \mathrm{Da}$, cysteine carbamidomethylation was considered as fixed modification, and oxidation of methionine and deamidation of asparagine/glutamine were considered as variable modifications. Trypsin was set as the proteolytic enzyme and one missed cleavage was allowed. During the MaxQuant search, a false discovery rate (FDR) of less than $1 \%$ was applied to increase the confidence of peptide and protein identification. To estimate FDR, a decoy protein database, generated from the human protein sequence database, was used. Proteins identified with only one unique peptide were discarded. Therefore, only proteins identified with at least two unique peptides and with an FDR less than $1 \%$ were used for further analysis.

Based on ion intensity, protein quantification was manually calculated using myoglobin as standard. From the three standards, myoglobin proved to be the one that generated reliable unique peptides. For quantification purposes, the three most intense tryptic peptide signals of the spike-in protein (myoglobin) and at least two most intense tryptic peptide signals of the proteins in the sample were primarily selected, as previously reported (Silva et al., 2006). To increase the precision of the quantification, peptides containing missed cleavages and posttranslational modifications were discarded.

Assessment of Protein Subcellular Localization, Molecular Function, and Composition of Skin Samples. To assess differences in the make-up of the human skin and LabSkin, the subcellular localization of all identified proteins $(n=$ 3030,1749 , and 1463, respectively) was annotated according to three databases: Gene Ontology, UniProt, and the Human Protein Atlas (HPA), by searching the gene names against these repositories. In addition, the PANTHER (Protein ANalysis THrough Evolutionary Relationships) classification system version 14.0 (http://www.pantherdb.org/) was used to assign protein functional class. Markers for different cell types in the skin epidermis (keratinocytes, Langerhans cells) and different sublayers (stratum spinosum, stratum granulosum, and stratum corneum) were identified using HPA and UniProt databases.

Statistical Analysis. All statistical analysis of the data was performed using Microsoft Excel 2010 and GraphPad Prism version 7.04 (La Jolla, CA). Abundance data of proteins in human skin samples are represented as means \pm S.D., medians, ranges, and coefficients of variation (CVs). The protein abundance measured from LabSkin 1 and 2 are presented as individual values. To assess similarity between ex vivo human skin and LabSkin, we evaluated whether relevant proteins quantified in human skin were present in LabSkin. Figures were generated using GraphPad Prism version 7.04.

\section{MALDI-Mass Spectrometry Imaging}

Sample Preparation. Terbinafine hydrochloride and tolbutamide were made up as $0.5 \%(\mathrm{w} / \mathrm{v})$ solutions in an emulsion of 80:20 (v/v) acetone:olive oil with $10 \%$ (v/v) isosorbide dimethyl ether and $20 \mu \mathrm{l}$ of each solution was topically applied on LabSkin in triplicate. Treated LabSkin was incubated at $37^{\circ} \mathrm{C}$ for 24 hours. Methylphenidate hydrochloride was made up as $0.5 \%(\mathrm{w} / \mathrm{v})$ solutions in an emulsion of 80:20 (v/v) water:olive oil with 10\% (v/v) isosorbide dimethyl ether, and $20 \mu \mathrm{l}$ of each solution was topically applied on LabSkin in triplicate. Treated LabSkin was incubated at $37^{\circ} \mathrm{C}$ for 24 hours. After incubation, LabSkin was washed with high-performance liquid chromatography-grade ethanol to remove excess formulation and then snap-frozen with liquid nitrogen cooled isopentane (2-5 minutes) and stored at $-80^{\circ} \mathrm{C}$. For cryosectioning, the LabSkin sections were transferred into a Cryostat (Leica 200 UV; Leica Microsystems, Milton Keynes, UK), mounted onto a cork ring using distilled water at $-25^{\circ} \mathrm{C}$ for 30 minutes to allow thermal equilibration. Tissue sections were cryosectioned at $12 \mu \mathrm{m}$, thaw mounted onto polylysine-coated glass slides for analysis on the Synapt G2 and Indium tin oxide slides for analysis using the Bruker Autoflex III, and stored at $-80^{\circ} \mathrm{C}$.

Sublimation. Three hundred milligrams of matrix $\alpha$-cyano-4-hydroxycinnamic acid was spread evenly at the bottom of the sublimation apparatus (Sigma Aldrich, Gillingham, UK). Polylysine glass slides contain LabSkin tissue sections were attached to the flat top of the chamber. The flat top of the chamber was then attached to the bottom using an O-ring seal, and vacuum was applied. When a stable vacuum of $2.5 \times 10^{-2}$ Torr was achieved, the top was filled with cold water $\left(5^{\circ} \mathrm{C}\right)$ and the temperature was set up at $180^{\circ} \mathrm{C}$. The sublimation process was performed until the optimal amount of $\alpha$-cyano-4-hydroxycinnamic acid (between 0.1 and $0.2 \mathrm{mg} \mathrm{cm}^{-2}$ ) was achieved. After sublimation, a recrystallization step was performed.

MALDI-Mass Spectrometry Imaging. Tissues sections of LabSkin treated with terbinafine hydrochloride and tolbutamide were imaged using a Bruker
Autoflex III fitted with a "Smartbeam" Nd:YAG laser $(355 \mathrm{~nm}, 1 \mathrm{kHz}$ ) (Bruker, Bremen, Germany) at a spatial resolution $50 \mu \mathrm{m} \times 50 \mu \mathrm{m}$, at a range of $100-1500 \mathrm{~m} / \mathrm{z}$. The tissues sections of LabSkin treated with methylphenidate hydrochloride were imaged using a Synapt G2 (Waters, Manchester, UK) in full scan sensitivity mode at a range of $100-1500 \mathrm{~m} / \mathrm{z}$ with a resolution 10,000 full width at half maximum at a spatial resolution of $60 \mu \mathrm{m} \times 60 \mu \mathrm{m}$, and with laser energy set to 250 arbitrary units. The ion mobility function of the instrument was not enabled. All images were acquired in positive ion mode. MALDI-mass spectrometry imaging (MSI) data for LabSkin tissue sections treated with terbinafine hydrochloride and tolbutamide were processed using the FlexAnalysis software (Bruker Daltonics). MALDI-MSI data for LabSkin tissue sections treated with methylphenidate hydrochloride were processed using High Definition Imaging (HDI) 1.4 mass spectrometry imaging software (Waters).

\section{Results}

A label-free global quantitative proteomic analysis of six human skin samples and two independent replicates of LabSkin samples was carried out, with a particular focus on the analysis of xenobiotic metabolizing enzymes and transporters (phases I, II, and III) and other enzymes (redox enzymes, peptidases and peptidases inhibitors, and endonucleases) that support xenobiotic metabolism in skin. We identified and quantified approximately 2000 proteins in both human skin and LabSkin from which 78 XMEs, including some auxiliary enzymes and transporters (Tables 2-4), 36 redox enzymes (Table 5), and 60 enzymes (Table 6) belonging to the peptidase family, nuclease family, and peptidases inhibitor family were quantified. MALDI imaging was used to validate the label-free proteomics data, particularly with regard to P450 and esterase presence.

Evaluation of Protein Subcellular Localization, Molecular Function, and Composition of Skin Samples. The number of proteins quantified in human skin was higher $(n=3030)$ than in LabSkin replicate $1(n=1749)$ (hereafter called LabSkin 1) and LabSkin replicate 2 ( $n=1463$ ) (hereafter called LabSkin 2). The localization and functional assignment of proteins identified in human skin samples and LabSkin replicates are shown in Fig. 1. Localization (Fig. 1A) and functional analysis (Fig. 1C) reflected differences between human skin and LabSkin, with an overlap of about $32 \%$ (Fig. 1B). An analysis of cellular markers was performed using the proteomics data. Human skin showed markers for both keratinocytes (calmodulin-like protein 5 (CALML5), keratin 19 (KRT19)) and Langerhans cells (CD207) whereas LabSkin contained markers for keratinocytes only. Markers for layers of epidermis were found in both human skin and LabSkin, including stratum spinosum markers (keratin 10 (KRT10), caspase 14 (CASP14)), stratum granulosum marker filaggrin (FLG) and stratum corneum marker kallikrein-related peptidase 5 (KLK5), although LabSkin 2 lacked detectable stratum corneum markers. A schematic representation of the different layers and cell types of the epidermis is shown in Fig. 2. In general, the abundance of all quantified markers were expressed at higher levels in human skin, followed by LabSkin 1 and then LabSkin 2 (Table 1).

Abundance of XME and Transporters. We were able to quantify a variety of proteins with known roles in phase I, phase II, and phase III drug/xenobiotic metabolism from the total cellular protein extract (Fig. 3). Fig. 3A and Table 2 provide quantitative information regarding phase I XME in human skin and LabSkin. The most abundant phase I XMEs in human skin sections were alcohol dehydrogenase 1B, ADH1B $\left(23.39 \pm 14.12 \mathrm{pmol} \mathrm{mg}^{-1}\right)$, alcohol dehydrogenase 1C, ADH1C $\left(37.46 \pm 15.05 \mathrm{pmol} \mathrm{mg}^{-1}\right)$, aldehyde dehydrogenase 1 , ALDH1A1 $\left(21.01 \pm 6.70 \mathrm{pmol} \mathrm{mg}^{-1}\right)$, and carbonyl reductase (CBR) $1(25.30 \pm$ 6.99 pmol $\left.\mathrm{mg}^{-1}\right)$. These enzymes were also quantified as highly abundant enzymes in LabSkin, although ADH1C and ALDH1A1 were absent in LabSkin 1. 
A

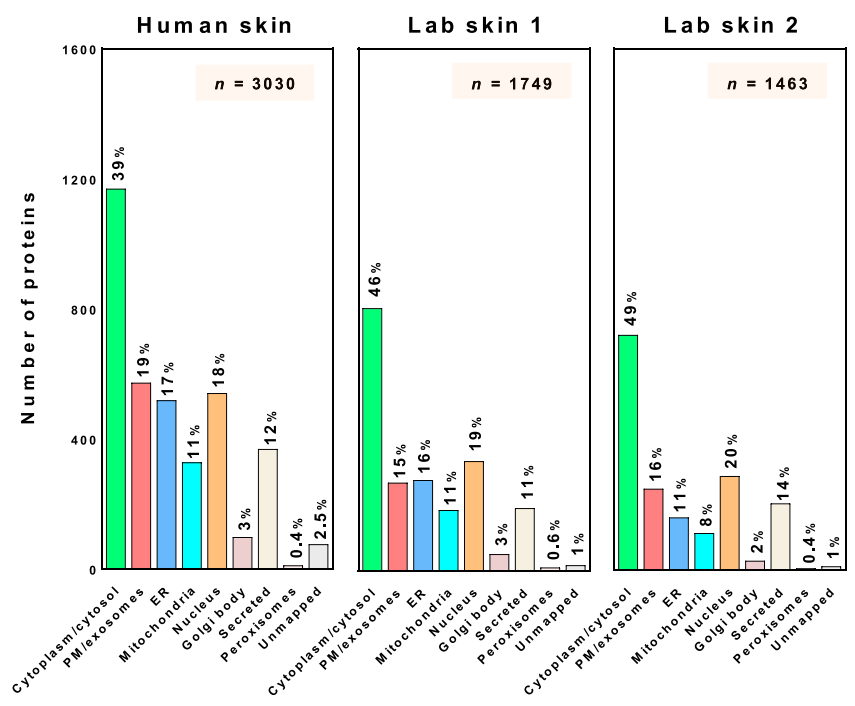

B

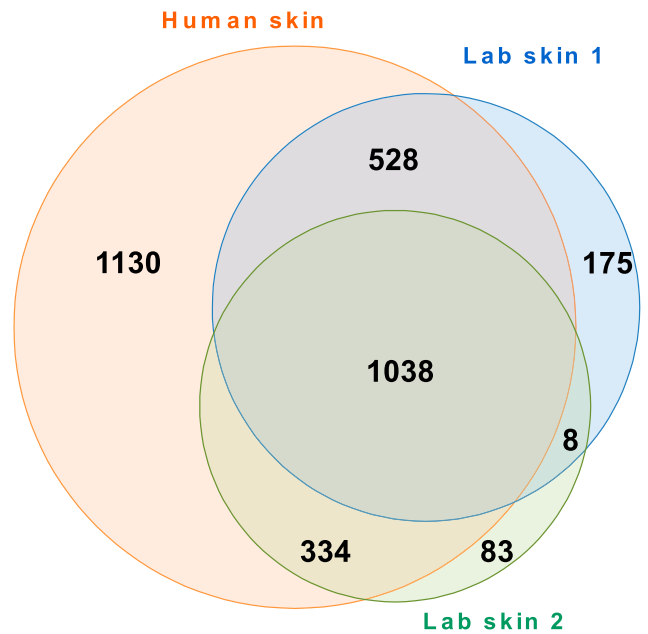

C

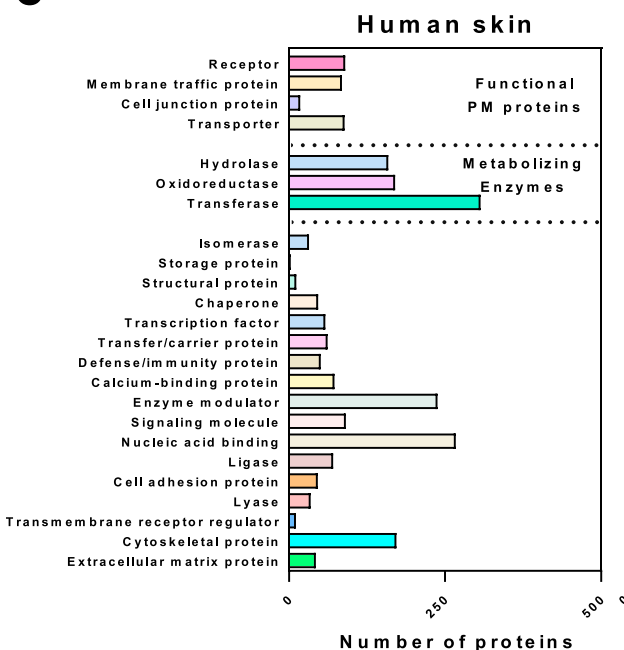

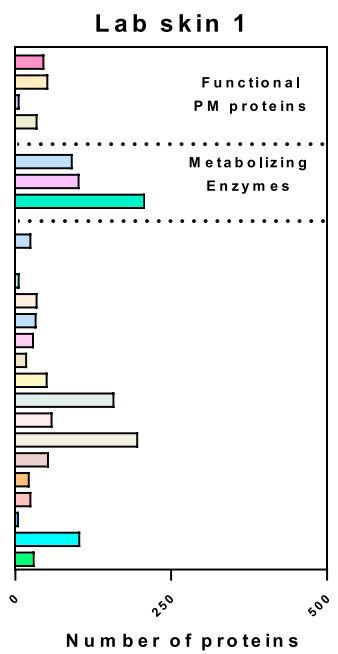
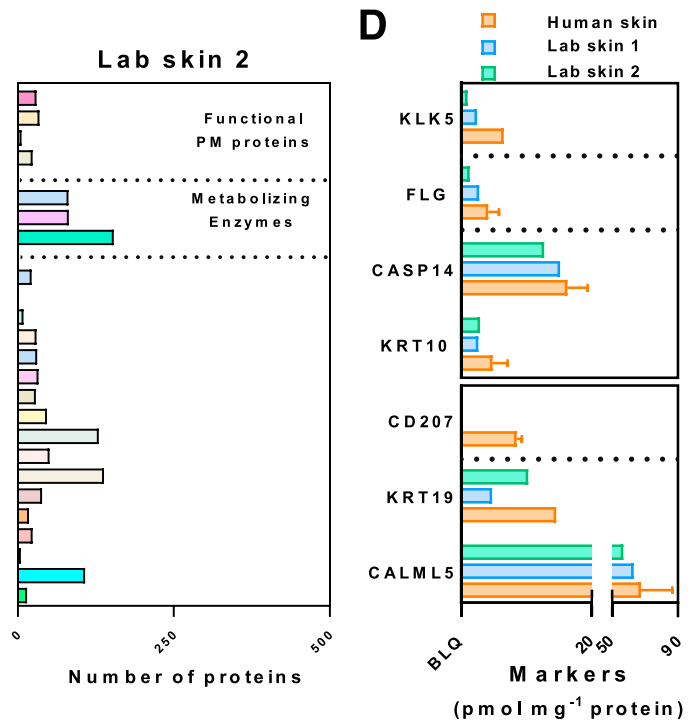

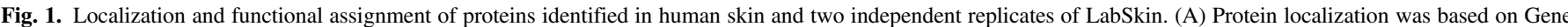

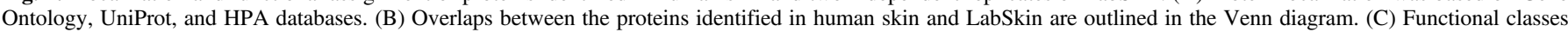

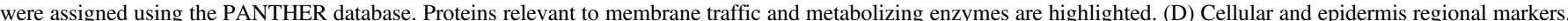

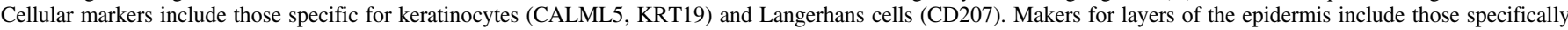

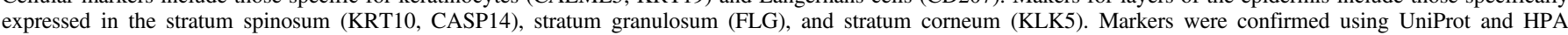
databases. ER, endoplasmic reticulum; $n$, number of proteins; PM, plasma membrane; BLQ, below the limit of quantification.

Cytochrome P450 enzymes are especially prevalent in the liver (Couto et al., 2019; El-Khateeb et al., 2019), and to a lesser extent the intestine (Couto et al., 2020). In the skin only three cytochrome P450 enzymes, namely, CYP51A1 (0.98 $\left.\pm 0.09 \mathrm{pmol} \mathrm{mg}^{-1}\right)$, CYP7B1 (2.57 $\left.\pm 0.45 \mathrm{pmol} \mathrm{mg}^{-1}\right)$, and CYP8A1 $\left(5.52 \pm 4.70 \mathrm{pmol} \mathrm{mg}^{-1}\right)$, were identified and quantified. These enzymes were also quantified in LabSkin 1, where they were present in approximately similar abundance to that in human skin, but not in LabSkin 2. A similar trend was observed with the auxiliary proteins for $\mathrm{P} 450 \mathrm{~s}$, including cytochrome b5, CYB5A $\left(9.54 \pm 5.15 \mathrm{pmol} \mathrm{mg}^{-1}\right)$, NADPH-cytochrome $\mathrm{P} 450$ reductase, POR $\left(4.46 \pm 0.77 \mathrm{pmol} \mathrm{mg}^{-1}\right)$, and several isoforms of NADH-cytochrome b5 reductase (CYB5R1/R2/R3). These proteins were only quantified in human skin and LabSkin 1 as shown in Table 2.

Three alcohol dehydrogenases and several aldehyde dehydrogenase isoforms were found in high abundance in human skin (Table 2). Alcohol dehydrogenases convert alcohols into aldehydes or ketones. Of the three alcohol dehydrogenases quantified, ADH1B and ADH5 were quantified in human skin and LabSkin. Although ADH5 has previously been reported in skin at the protein level, ADH1B has previously been observed only as mRNA (Uhlen et al., 2015). In addition to these, $\mathrm{ADH} 1 \mathrm{C}$ was quantified for the first time in human skin and in LabSkin 2. Aldehyde dehydrogenases are involved in the oxidation of a plethora of aldehyde groups to carboxylates and were expressed with similar abundance in LabSkin and human skin. We have quantified ALDH1L2 in human skin at the protein level for the first time, although the corresponding mRNA level has previously been observed (Uhlen et al., 2015).

A number of reductases were quantified including several isoforms of the aldo-keto reductase family, two carbonyl reductase isoforms and biliverdin reductase A (BLVRA). The aldo-keto reductases AKR1A1, AKR1B1, and AKR1C2 have previously been reported in human skin only at the level of mRNA, whereas AKR1D1 has not previously been reported in any form (Uhlen et al., 2015). BLVRA and the carbonyl reductases were found at similar levels in LabSkin compared with 


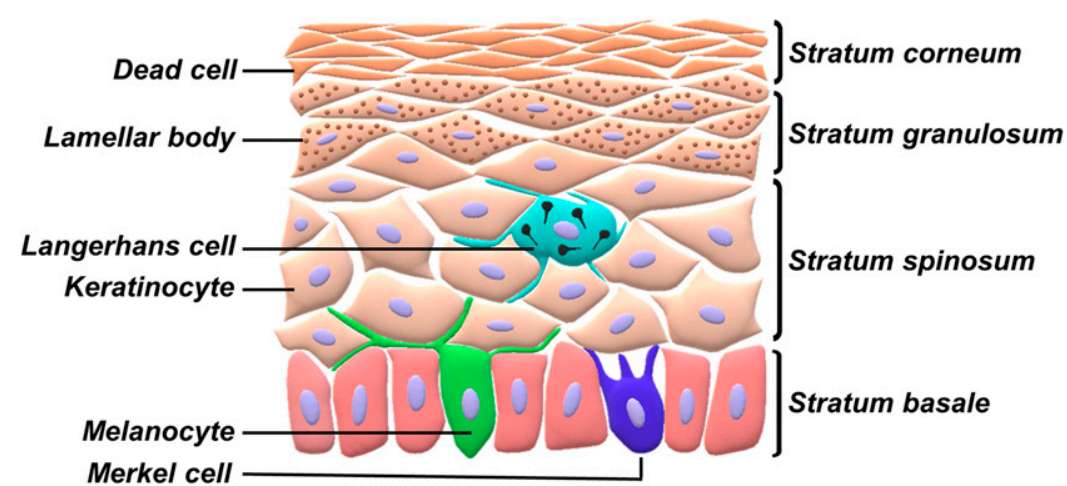

Fig. 2. Schematic representation of the layers of thin human skin epidermis. From the deep to the superficial layer, the epidermis consists of stratum basale, stratum spinosum, stratum granulosum, and stratum corneum. In thick human skin, a discernible fifth layer (not represented here) called stratum lucidum is present between the stratum granulosum and stratum corneum. Epidermis is mostly composed of keratinocytes. Melanocytes and Merkel cells are present in low abundance in the stratum basale, and Langerhans cells are predominantly present in low abundance in the stratum spinosum. human skin, whereas the aldo-keto reductases were predominantly expressed at lower levels.

Other phase I enzymes quantified are shown in Table 2. Examples include amine oxidase (AOC) and AOC3, aldehyde oxidase 1, carboxylesterase 1 (CES1), epoxide hydrolase (EPHX) 1/2, and leukotriene A4 hydrolase. In general ( $80 \%$ of the quantified other phase I enzymes) human skin and LabSkin 1 showed similar levels of these enzymes, but the abundances of a large proportion of these proteins (50\%) were lower in LabSkin 2 compared with human skin. AOC2,

\section{TABLE 1}

Expression levels of cellular markers including those specific for keratinocytes and Langerhans cells

Identified makers for layers of the epidermis include proteins specifically expressed in the stratum spinosum, stratum granulosum, and stratum corneum. Protein identification and quantification was based on the presence of at least two unique peptides and from human skin in at least two donors. Protein expression is represented by the mean, the S.D., and the percent $\mathrm{CV}$. The range of protein expression (min-max) and the number of samples (n) are also reported. Protein abundance is reported in picomoles milligram ${ }^{-1}$ of protein.

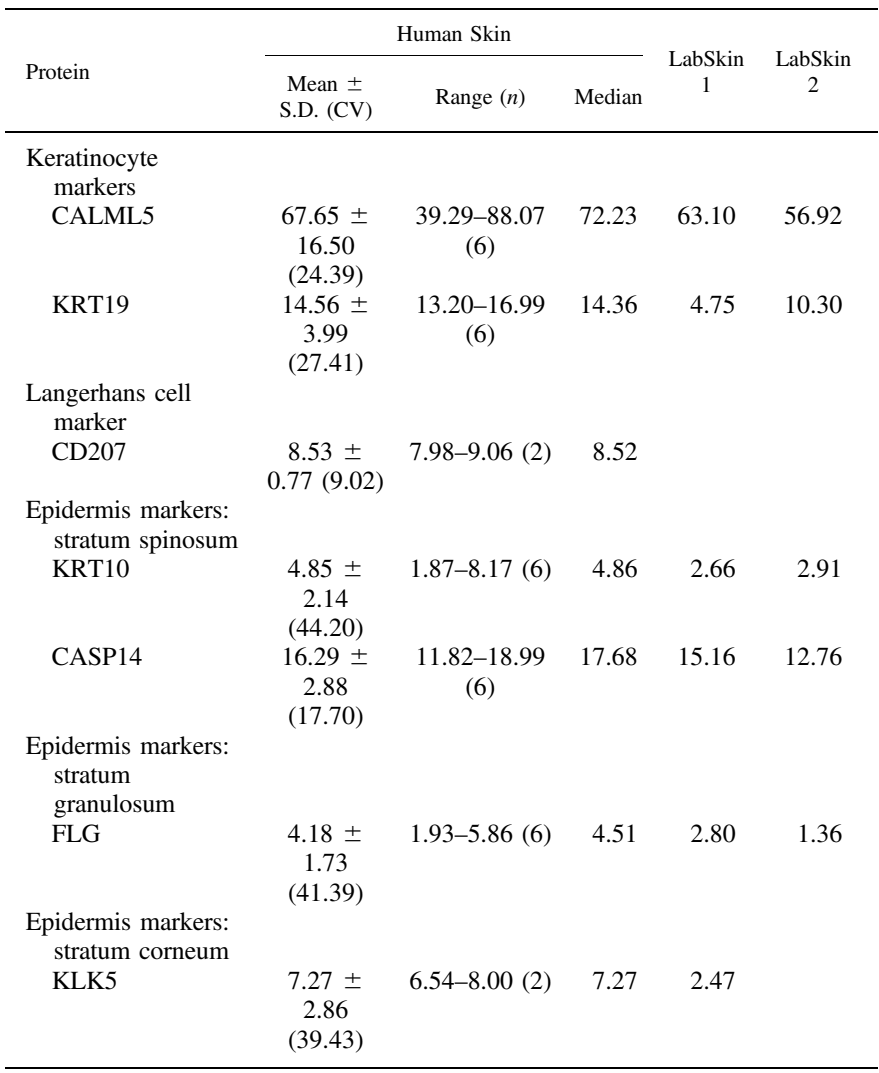

monoamine oxidase A (MAOA), and vitamin $\mathrm{K}$ epoxide reductase complex subunit 1 (VKORC1) were quantified in human skin but not in LabSkin.

The phase II XME identified and quantified in the human skin and LabSkin are shown in Fig. 3B and Table 3. These include glutathione S-transferases, sulfotransferases, and other transferases. In contrast with the human liver, no UGT enzymes were identified in these samples indicating that glucuronidation plays a minor role in skin, whereas glutathionylation and sulfonation are important reactions in the metabolism of several xenobiotics and drugs (Couto et al., 2019). From all phase II enzymes identified and quantified, $\operatorname{GSTP} 1(\pi)$ is the most abundant with concentration $62.65 \pm 17.78 \mathrm{pmol} \mathrm{mg}^{-1}$ protein, followed by GSTM4 $(\mu)$ and GSTM3 $(\mu)$ with concentrations of $21.75 \pm 0.63$ and $19.68 \pm 10.29 \mathrm{pmol} \mathrm{mg}^{-1}$ protein, respectively. These three enzymes have previously been reported only at the level of mRNA (Uhlen et al., 2015). Among glutathione S-transferases, $45 \%$ of the enzymes (four out of nine) quantified in human skin were quantified in LabSkin 1, and $78 \%$ of the enzymes (seven out of nine) quantified in the human skin were also quantified in LabSkin 2. LabSkin 2 contains almost all the glutathione S-transferases found in skin, but often at reduced concentrations. In LabSkin 1, fewer glutathione S-transferases were detected, but their concentrations fell within the range found in human skin. In stark contrast to glutathione S-transferases, sulfotransferases were not detected in LabSkin despite the quantification of these enzymes in the human skin sections. The occurrence of S-adenosylmethionine synthase isoform type-1A (MAT1A) (5.36 $\left.\pm 1.87 \mathrm{pmol} \mathrm{mg}^{-1}\right)$ is a new finding, not previously reported in human skin (Uhlen et al., 2015). Among the seven other transferases detected in the human skin, four were quantified in LabSkin 1 and six were quantified in LabSkin 2. The abundance of two proteins, catechol-O-methyltransferase (COMT) and NNMT, was higher in LabSkin 1 compared with human skin. The abundance of all six quantified proteins in LabSkin 2 was within the range quantified in human skin. Thiopurine S-methyltransferase was below the limit of quantification in LabSkin, and this enzyme was only quantified in two out of six human skin samples.

Only a few phase III transporters were found in our skin samples, and they were generally of low abundance, as shown in Fig. $3 \mathrm{C}$ and Table 4. Of the $\mathrm{ABC}$ transporters, only $\mathrm{ABCA} 8$ and $\mathrm{ABCB} 11$ were securely quantified in the human skin, but $\mathrm{ABCA} 8$ could not be detected in LabSkin. Among the eight SLC transporters quantified in human skin, fewer than $50 \%$ were quantified in LabSkin, and they were generally found in lower abundance compared with human skin.

Assessment of the Abundance of Enzymes with a Supportive Role in Xenobiotic Metabolism: Redox Enzymes. Phase II XME are typically involved in detoxification of a wide variety of highly reactive intermediate substrates formed in phase I. Therefore, redox enzymes 
A

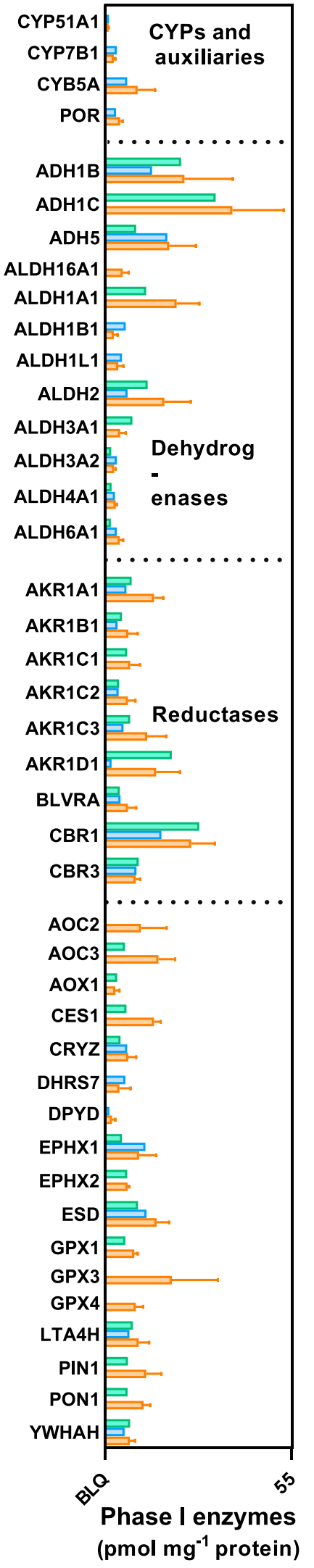

$\mathbf{B}$

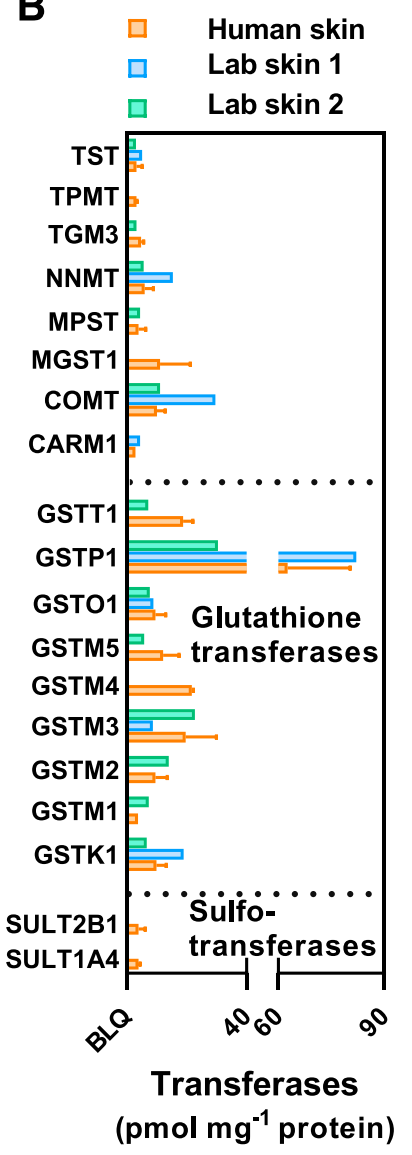

Fig. 3. The protein quantities of epidermal enzymes and transporters expressed in human skin and LabSkin. (A) Phase I enzymes include cytochrome P450 monooxygenases, dehydrogenases, reductases, and hydrolases. (B) Phase II enzymes are involved in conjugation reactions and include glutathione S-transferases and sulfotransferases. (C) Phase III proteins are transporters that include ABC transporters and SLCs. Data are presented as means and S.D. (error bars) for human skin samples $(n=6)$ and individual values for LabSkin 1 and 2. BLQ, below the limit of quantification.

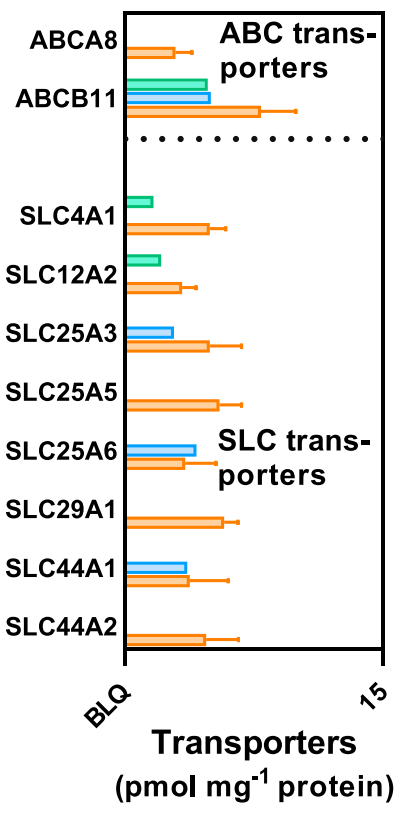


TABLE 2

Expression levels of phase I xenobiotic metabolizing enzymes in human skin and LabSkin

Protein identification and quantification was based on the presence of at least two different peptides and from human skin of at least two donors. Protein expression is represented by the mean, the

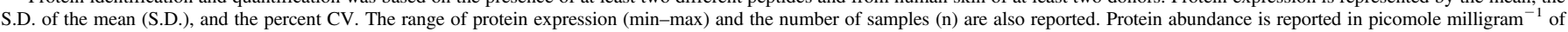
protein.

\begin{tabular}{|c|c|c|c|c|c|}
\hline \multirow{2}{*}{ Protein } & \multicolumn{3}{|c|}{ Human Skin } & \multirow{2}{*}{ LabSkin 1} & \multirow{2}{*}{ LabSkin 2} \\
\hline & Mean \pm S.D. $(C V)$ & Range $(n)$ & Median & & \\
\hline \multicolumn{6}{|c|}{ Cytochrome $\mathrm{P} 450$ and auxiliary proteins } \\
\hline CYP51A1 ${ }^{\mathrm{a}}$ & $0.98 \pm 0.09(9.38)$ & $0.92-1.05(2)$ & 0.98 & 1.19 & \\
\hline CYP7B1 & $2.57 \pm 0.45(17.43)$ & $2.26-2.89(2)$ & 2.57 & 3.50 & \\
\hline CYP8A1 (PTGIS) & $5.52 \pm 4.70(91.25)$ & $1.58-12.73(4)$ & 3.88 & 2.60 & \\
\hline POR & $4.46 \pm 0.77(17.16)$ & $3.66-5.19(3)$ & 4.54 & 3.21 & \\
\hline CYB5A & $9.54 \pm 5.15(54.02)$ & $3.26-15.45(6)$ & 10.56 & 6.52 & \\
\hline CYB5R1 & $11.31 \pm 6.05(53.55)$ & $7.02-15.59(2)$ & 11.31 & 2.06 & \\
\hline CYB5R2 $2^{\mathrm{a}}$ & $5.93 \pm 2.02(34.00)$ & $3.5-8.58(5)$ & 5.31 & 3.55 & \\
\hline CYB5R3 & $10.27 \pm 2.57(25.05)$ & $6.98-13.05(6)$ & 10.66 & 20.75 & \\
\hline \multicolumn{6}{|l|}{ Dehydrogenases } \\
\hline $\mathrm{ADH} 1 \mathrm{~B}^{\mathrm{a}}$ & $23.39 \pm 14.12(60.39)$ & $4.01-48.02(6)$ & 21.60 & 13.86 & 22.40 \\
\hline $\mathrm{ADH} 1 \mathrm{C}^{\mathrm{b}}$ & $37.46 \pm 15.05(40.16)$ & $13.80-55.35(5)$ & 39.01 & & 32.53 \\
\hline ADH5 & $18.81 \pm 7.96(42.32)$ & $4.73-29.04(6)$ & 19.38 & 18.30 & 9.08 \\
\hline ALDH16A1 & $5.23 \pm 1.74(33.32)$ & $3.41-6.88(3)$ & 5.39 & & \\
\hline ALDH1A1 & $21.01 \pm 6.70(31.86)$ & $11.88-28.84(5)$ & 21.35 & & 12.13 \\
\hline ALDH1B1 & $2.59 \pm 1.09(42.19)$ & $1.71-3.82(5)$ & 1.90 & 6.09 & \\
\hline ALDH1L1 & $3.93 \pm 1.53(38.85)$ & $2.40-5.45(3)$ & 3.95 & 4.93 & \\
\hline $\mathrm{ALDH}_{1 \mathrm{~L} 2}{ }^{\mathrm{a}}$ & $4.09 \pm 1.52(37.27)$ & $3.01-5.17(2)$ & 4.09 & 7.55 & \\
\hline ALDH2 & $17.48 \pm 7.75(44.32)$ & $5.31-25.01(6)$ & 19.97 & 6.64 & 12.54 \\
\hline ALDH3A1 & $4.45 \pm 1.60(36.01)$ & $2.76-6.12(5)$ & 4.56 & & 8.08 \\
\hline ALDH3A2 & $2.67 \pm 0.43(15.93)$ & $2.22-3.28(6)$ & 2.55 & 3.39 & 1.77 \\
\hline ALDH4A1 & $3.10 \pm 0.33(10.78)$ & $2.72-3.31(3)$ & 3.28 & 2.95 & 1.97 \\
\hline ALDH6A1 & $4.27 \pm 1.07(25.06)$ & $2.48-5.82(6)$ & 4.33 & 3.46 & 1.65 \\
\hline BDH2 & $6.13 \pm 3.46(56.51)$ & $3.68-8.58(2)$ & 6.13 & 3.03 & \\
\hline \multicolumn{6}{|l|}{ Reductases } \\
\hline $\mathrm{AKR} 1 \mathrm{~A} 1^{\mathrm{a}}$ & $14.39 \pm 2.68(18.65)$ & $11.39-17.98(6)$ & 14.43 & 6.30 & 7.82 \\
\hline $\mathrm{AKR} 1 \mathrm{~B} 1^{\mathrm{a}}$ & $6.87 \pm 2.74(39.85)$ & $2.59-9.66(5)$ & 6.89 & 3.63 & 4.93 \\
\hline AKR1C1 & $7.35 \pm 2.91(39.57)$ & $4.21-11.62(6)$ & 6.73 & & 6.53 \\
\hline $\mathrm{AKR}_{1 C} 2^{\mathrm{a}}$ & $6.70 \pm 2.17(32.32)$ & $3.90-9.31(6)$ & 6.33 & 4.03 & 4.11 \\
\hline AKR1C3 & $12.42 \pm 5.60(45.07)$ & $4.41-18.98(6)$ & 14.11 & 5.41 & 7.37 \\
\hline AKR1D $1^{b}$ & $15.03 \pm 7.06(46.99)$ & $7.52-25.89(5)$ & 13.12 & 1.90 & 19.60 \\
\hline AKR7A2 & $8.01 \pm 1.32(16.51)$ & $7.08-8.95(2)$ & 8.01 & 6.88 & \\
\hline BLVRA & $6.75 \pm 2.34(34.70)$ & $3.92-9.36(5)$ & 6.12 & 4.56 & 4.27 \\
\hline CBR1 & $25.30 \pm 6.99(27.62)$ & $12.44-31.51(6)$ & 27.96 & 16.56 & 27.73 \\
\hline CBR3 & $9.00 \pm 1.32(14.62)$ & $7.45-10.44(5)$ & 9.59 & 9.20 & 9.93 \\
\hline \multicolumn{6}{|l|}{ Others } \\
\hline $\mathrm{AOC} 2^{\mathrm{a}}$ & $10.59 \pm 7.54(71.21)$ & $3.49-21.26(4)$ & 8.81 & & \\
\hline $\mathrm{AOC}^{\mathrm{a}}$ & $15.72 \pm 4.86(30.94)$ & $11.20-23.83(6)$ & 14.22 & & 5.89 \\
\hline AOX1 & $3.00 \pm 1.18(39.26)$ & $1.65-3.84(3)$ & 3.52 & & 3.55 \\
\hline $\mathrm{CES} 1^{\mathrm{a}}$ & $14.43 \pm 1.91(13.23)$ & $11.70-16.21(5)$ & 14.73 & & 6.29 \\
\hline CES1P1 $1^{\mathrm{b}}$ & $16.32 \pm 6.34(38.87)$ & $12.27-27.45$ & 14.37 & 3.07 & 10.52 \\
\hline CRYZ & $6.89 \pm 2.20(31.90)$ & $2.43-8.08(6)$ & 7.70 & 6.46 & 4.49 \\
\hline DHRS7 & $4.16 \pm 3.36(80.81)$ & $0.92-7.62(3)$ & 3.93 & 5.93 & \\
\hline DPYD $^{\mathrm{a}}$ & $1.98 \pm 1.06(53.73)$ & $0.94-3.07(3)$ & 1.94 & 1.28 & \\
\hline $\mathrm{EPHX} 1^{\mathrm{a}}$ & $10.00 \pm 5.06(50.63)$ & $4.17-15.57(6)$ & 9.91 & 11.84 & 4.97 \\
\hline EPHX2 & $6.57 \pm 0.51(7.73)$ & $6.21-6.93(2)$ & 6.57 & & 6.49 \\
\hline ESD & $15.15 \pm 3.72(24.58)$ & $9.29-18.38(6)$ & 17.00 & 12.18 & 9.69 \\
\hline LTA4H & $9.85 \pm 3.07(31.16)$ & $5.00-14.29(6)$ & 9.57 & 7.15 & 8.13 \\
\hline $\mathrm{PIN}^{\mathrm{a}}$ & $12.10 \pm 4.51(37.26)$ & $8.25-18.63(4)$ & 10.77 & & 6.71 \\
\hline PON1 & $11.28 \pm 1.99(17.64)$ & $8.32-13.81(5)$ & 11.20 & & 6.61 \\
\hline YWHAH & $7.23 \pm 1.57(21.67)$ & $4.92-9.00(6)$ & 7.08 & 5.69 & 7.33 \\
\hline MAOA & $7.16 \pm 7.03(98.27)$ & $2.18-12.13(2)$ & 7.16 & & \\
\hline PTGES $^{c}$ & $17.62 \pm 4.70(26.69)$ & $12.87-21.73(4)$ & 17.95 & 8.95 & \\
\hline PTGS1 (COX-1) & $3.93 \pm 0.06(1.41)$ & $3.89-3.97(2)$ & 3.93 & 10.88 & \\
\hline VKORC $1^{d}$ & $12.14 \pm 13.00(107.12)$ & $2.94-21.34(2)$ & 12.14 & & \\
\hline
\end{tabular}

AOX1, aldehyde oxidase 1; COX, cyclooxygenase; LTA4H, leukotriene A4 hydrolase.; ESD, esterase D; MAOA; monoamine oxidase A; PON1, serum paraoxonase/arylesterase 1; VKORC1, vitamin $\mathrm{K}$ epoxide reductase complex subunit 1 .

${ }^{a}$ According to Human Protein Atlas, these enzymes are reported to be present in the skin at the RNA level (low copy numbers) but not at the protein level.

${ }^{b}$ According to Human Protein Atlas, these enzymes are not reported to be present in the skin at the RNA or protein level.

${ }^{c}$ This is a nuclear receptor.

${ }^{d}$ Warfarin targets this enzyme therefore interfering with blood coagulation in humans.

such as catalase, glutathione synthetase, and peroxiredoxins are typically categorized as phase II XME (van Eijl et al., 2012). As shown in Table 5, human skin and LabSkin are very rich in redox enzymes. We identified and quantified a plethora of redox enzymes such as catalase, superoxide dismutases, thioredoxins, and glutaredoxins. Thioredoxin (TXN) was the most abundant in human skin $\left(84.87 \pm 20.01 \mathrm{pmol} \mathrm{mg}^{-1}\right)$ followed by peroxiredoxin (PRDX) $1\left(53.52 \pm 11.78 \mathrm{pmol} \mathrm{mg}^{-1}\right)$. Both were found in high abundance in both LabSkin replicates. Also highly abundant in both human skin and LabSkin $\left(>20 \mathrm{pmol} \mathrm{mg}^{-1}\right)$ were superoxide dismutase (SOD) 1/2/3, PRDX2/5/6, prolyl 4-hydroxylase, 
TABLE 3

Expression levels of phase II xenobiotic metabolizing enzymes in human skin and LabSkin

Protein identification and quantification was based on the presence of at least two different peptides and from human skin of at least two donors. Protein expression is represented by the mean, the

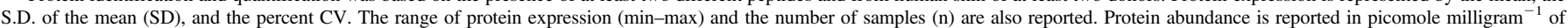
protein.

\begin{tabular}{|c|c|c|c|c|c|}
\hline \multirow{2}{*}{ Protein } & \multicolumn{3}{|c|}{ Human Skin } & \multirow{2}{*}{ LabSkin 1} & \multirow{2}{*}{ LabSkin 2} \\
\hline & Mean \pm S.D. $(\mathrm{CV})$ & Range $(n)$ & Median & & \\
\hline \multicolumn{6}{|c|}{ Glutathione transferases } \\
\hline GSTK1 (kappa) & $9.99 \pm 3.26(32.67)$ & $6.47-15.24(6)$ & 9.05 & 18.99 & 6.73 \\
\hline GSTM2 (mu) & $9.59 \pm 4.07(42.47)$ & $2.47-14.72(6)$ & 10.00 & & 14.11 \\
\hline $\operatorname{GSTM} 3(\mathrm{mu})^{\mathrm{a}}$ & $19.68 \pm 10.29(52.29)$ & $4.91-31.93(6)$ & 22.90 & 8.83 & 22.90 \\
\hline GSTM4 (mu) & $21.75 \pm 0.63(2.91)$ & $21.28-22.47(3)$ & 21.51 & & \\
\hline GSTM5 $(\mathrm{mu})^{\mathrm{a}}$ & $12.24 \pm 5.33(43.54)$ & $6.59-19.44(4)$ & 11.47 & & 5.94 \\
\hline GSTO1 (omega) & $9.59 \pm 3.43(35.72)$ & $4.75-13.71(6)$ & 9.04 & 8.94 & 7.83 \\
\hline GSTP1 (pi) & $62.65 \pm 17.78(28.38)$ & $39.31-82.46(6)$ & 60.22 & 82.10 & 30.45 \\
\hline GSTT1 (theta) & $18.84 \pm 3.31(17.56)$ & $16.50-21.17(3)$ & 18.84 & & 7.31 \\
\hline MGST1 & $11.26 \pm 9.94(88.27)$ & $2.69-22.16(3)$ & 8.93 & & \\
\hline \multicolumn{6}{|l|}{ Sulfotransferases } \\
\hline SULT1A $4^{\mathrm{a}}$ & $4.08 \pm 0.40(9.73)$ & $3.84-4.54(3)$ & 3.87 & & \\
\hline SULT2B1 & $3.98 \pm 2.21(55.61)$ & $2.42-5.55(2)$ & 3.98 & & \\
\hline \multicolumn{6}{|l|}{ Other transferases } \\
\hline COMT & $10.13 \pm 2.84(28.06)$ & $5.31-13.25(6)$ & 10.58 & 29.74 & 11.12 \\
\hline MAT1A $^{b}$ & $5.36 \pm 1.87(34.97)$ & $2.19-7.71(6)$ & 5.48 & 4.25 & 4.57 \\
\hline MPST & $3.97 \pm 2.44(61.52)$ & $1.21-5.86(3)$ & 4.84 & & 4.45 \\
\hline NNMT & $6.01 \pm 3.03(50.43)$ & $2.74-8.73$ & 6.57 & 15.47 & 5.65 \\
\hline TGM3 & $4.83 \pm 0.95(19.73)$ & $3.81-5.70(3)$ & 4.99 & & 3.35 \\
\hline TPMT & $3.27 \pm 0.22(6.73)$ & $3.11-3.42(2)$ & 3.27 & & \\
\hline TST & $3.36 \pm 1.81(53.86)$ & $1.66-6.44$ (5) 6.446 .44 & 2.82 & 5.20 & 3.08 \\
\hline
\end{tabular}

TPMT, thiopurine S-methyltransferase; MAT1A, S-adenosylmethionine synthase isoform type-1.

${ }^{a}$ According to Human Protein Atlas, these enzymes are reported to be present in the skin at the RNA level (low copy numbers) but not at the protein level

${ }^{b}$ According to Human Protein Atlas, these enzymes are not reported to be present in the skin at the RNA or protein level.

and protein disulfide-isomerase A (PDIA) 3/6. Among GPX enzymes, GPX3 enzyme was the most abundant in human skin $(19.57 \pm 13.60$ pmol mg ${ }^{-1}$ ), but GPX3/4 was absent in LabSkin. All three naturally found SOD isoforms were identified and quantified in human skin, and their abundance followed the rank order SOD3 $>$ SOD1 $>$ SOD2. SOD3 is also known as extracellular superoxide dismutase [Cu-Zn], and its abundance in human skin was in the range of $40.85 \pm 19.72 \mathrm{pmol}$ $\mathrm{mg}^{-1}$ protein. Although all SOD isoforms were found in LabSkin, their abundance and rank order were different to those found in human skin. We also identified and quantified the seleno proteins GPX4 (8.97 \pm 2.24 pmol mg ${ }^{-1}$ protein) and TXNRD1 (4.61 $\pm 1.51 \mathrm{pmol} \mathrm{mg}^{-1}$ protein) known for their important antioxidant role in the human skin. These enzymes have been reported to be the two most abundant seleno proteins expressed in the skin epidermis and cultured keratinocytes (Sengupta et al., 2010).

Assessment of the Abundance of Enzymes with a Supportive Role in Xenobiotic Metabolism: Peptidases and Nucleases. Proteolytic enzymes and their inhibitors are highly represented in skin, and they represent more than $2 \%$ of the human genome (Puente et al., 2005). Therefore, it is not surprising that proteases/peptidases and their inhibitors have an essential role in controlling multiple biologic processes, including xenobiotic metabolism (Craik et al., 2011). Proteases and protease inhibitors work together to control development and maturation of keratinocytes in the epidermis (Zeeuwen, 2004). Proteases from the serine, metallo, aspartic, and cysteine categories were identified and quantified in both human skin and LabSkin, as shown in

TABLE 4

Expression levels of phase III xenobiotic transporters in human skin and LabSkin

Protein identification and quantification was based on the presence of at least two different peptides and from human skin of at least two donors. Protein expression is represented by the mean, the S.D. of the mean (S.D.), the percent CV, the range (min-max), and the number of samples (n). Protein abundance is reported in picomole milligram ${ }^{-1}$ of protein.

\begin{tabular}{|c|c|c|c|c|c|}
\hline \multirow{2}{*}{ Protein } & \multicolumn{3}{|c|}{ Human Skin } & \multirow{2}{*}{ LabSkin 1} & \multirow{2}{*}{ LabSkin 2} \\
\hline & Mean \pm S.D. $(\mathrm{CV})$ & Range $(n)$ & Median & & \\
\hline \multicolumn{6}{|c|}{ ATP-binding cassette } \\
\hline ABCA8 & $2.92 \pm 0.97(33.10)$ & $2.24-3.60(2)$ & 2.92 & & \\
\hline $\mathrm{ABCB} 11^{\mathrm{a}}$ & $7.90 \pm 2.03(25.64)$ & $5.39-10.05(4)$ & 8.09 & 4.99 & 4.81 \\
\hline \multicolumn{6}{|l|}{ Solute carriers } \\
\hline SLC12A2 & $3.31 \pm 0.80(24.24)$ & $2.43-4.52(5)$ & 3.07 & & 2.10 \\
\hline SLC $25 \mathrm{~A} 3^{\mathrm{b}}$ & $4.92 \pm 1.85(37.65)$ & $2.98-7.23(4)$ & 4.74 & 2.84 & \\
\hline SLC25A5 & $5.50 \pm 1.27(23.00)$ & $4.61-6.40(2)$ & 5.50 & & \\
\hline SLC25A6 & $3.50 \pm 1.79(51.19)$ & $1.46-4.82(3)$ & 4.21 & 4.16 & \\
\hline SLC29A1 & $5.77 \pm 0.79(13.70)$ & $5.21-6.32(2)$ & 5.77 & & \\
\hline SLC44A $1^{\text {b }}$ & $3.75 \pm 2.24(59.81)$ & $2.29-6.34(3)$ & 2.63 & 3.62 & \\
\hline SLC44A2 ${ }^{\mathrm{b}}$ & $4.71 \pm 1.89(40.22)$ & $3.37-6.04(2)$ & 4.71 & & \\
\hline SLC4A $1^{\mathrm{a}}$ & $4.94 \pm 0.92(18.64)$ & $4.22-6.20(4)$ & 4.67 & & 1.65 \\
\hline
\end{tabular}

${ }^{a}$ According to Human Protein Atlas, these transporters are not reported to be present in the skin at the RNA or protein level.

${ }^{b}$ According to Human Protein Atlas, these transporters are reported to be present in the skin at the RNA level (low copy numbers) but not at the protein level. 
TABLE 5

Expression levels of enzymes in human skin and LabSkin with a role in oxidative stress and xenobiotic metabolism

Protein identification and quantification was based on the presence of at least two different peptides and from human skin of at least two donors. Protein expression is represented by the mean, the S.D. of the mean (S.D.), the percent CV, the range (min-max), and the number of samples (n). Protein abundance is reported in picomole milligram ${ }^{-1}$ of protein.

\begin{tabular}{|c|c|c|c|c|c|}
\hline \multirow{2}{*}{ Protein } & \multicolumn{3}{|c|}{ Human Skin } & \multirow{2}{*}{ LabSkin 1} & \multirow{2}{*}{ LabSkin 2} \\
\hline & Mean \pm S.D. $(\mathrm{CV})$ & Range $(n)$ & Median & & \\
\hline \multicolumn{6}{|l|}{ Catalase } \\
\hline CAT $^{\mathrm{a}}$ & $13.63 \pm 4.38(32.15)$ & $7.78-18.40(6)$ & 14.30 & 6.37 & 8.49 \\
\hline \multicolumn{6}{|c|}{ Superoxide dismutases } \\
\hline SOD1 & $24.99 \pm 5.16(20.66)$ & $19.61-31.52(6)$ & 23.36 & 12.35 & 33.59 \\
\hline SOD2 & $22.99 \pm 15.88(69.07)$ & $10.48-54.23(6)$ & 19.53 & 67.11 & 13.45 \\
\hline SOD3 & $40.85 \pm 19.72(48.28)$ & $6.22-62.05(6)$ & 42.87 & 9.29 & 20.39 \\
\hline $\mathrm{CCS}$ & $3.61 \pm 1.04(28.73)$ & $2.88-5.08(4)$ & 3.24 & & 2.56 \\
\hline \multicolumn{6}{|c|}{ Glutathione peroxidases } \\
\hline GPX1 & $8.59 \pm 0.96(11.12)$ & $7.56-10.04(5)$ & 8.69 & & 5.99 \\
\hline GPX3 & $19.57 \pm 13.60(69.50)$ & $7.32-38.04(5)$ & 13.28 & & \\
\hline GPX4 & $8.97 \pm 2.24(24.96)$ & $6.61-12.08(5)$ & 9.01 & & \\
\hline \multicolumn{6}{|l|}{ Glutaredoxins } \\
\hline GLRX $^{\mathrm{a}}$ & $16.93 \pm 6.71(39.62)$ & $11.70-24.49(3)$ & 14.59 & 12.39 & 6.36 \\
\hline GLRX3 & $3.92 \pm 0.13(3.26)$ & $3.83-4.01(2)$ & 3.92 & & 2.58 \\
\hline \multicolumn{6}{|l|}{ Thioredoxins } \\
\hline TXNRD1 & $4.61 \pm 1.51(32.84)$ & $3.53-6.81(4)$ & 4.05 & 1.43 & 5.41 \\
\hline $\mathrm{TXN}$ & $84.87 \pm 20.01(23.58)$ & $61.22-112.57(6)$ & 86.32 & 59.33 & 80.07 \\
\hline TXN2 & $7.68 \pm 2.77(36.10)$ & $4.13-11.07(5)$ & 7.52 & & 5.51 \\
\hline TXNL1 & $6.84 \pm 2.16(31.59)$ & $3.39-9.65(6)$ & 6.89 & 3.83 & 4.65 \\
\hline TXNDC5 & $9.50 \pm 2.07(21.82)$ & $6.83-12.48(6)$ & 9.25 & 23.71 & 6.87 \\
\hline TXNDC $12^{\mathrm{a}}$ & $3.95 \pm 0.52(13.06)$ & $3.36-4.58(4)$ & 3.93 & 3.01 & \\
\hline TXNDC17 & $11.56 \pm 3.09(26.70)$ & $7.78-16.83(6)$ & 11.09 & 14.28 & 8.88 \\
\hline TMX1 & $11.48 \pm 1.75(15.25)$ & $10.24-12.71(2)$ & 11.48 & 7.16 & \\
\hline \multicolumn{6}{|c|}{ Glutathione metabolism } \\
\hline GSR & $8.74 \pm 2.16(24.70)$ & $5.36-11.35(5)$ & 9.06 & 4.89 & 5.03 \\
\hline GSS & $6.33 \pm 1.93(30.57)$ & $4.32-9.07(6)$ & 6.06 & 5.99 & 3.79 \\
\hline HAGH & $3.52 \pm 1.99(56.66)$ & $1.78-6.31(4)$ & 3.00 & & \\
\hline $\mathrm{GLO}^{\mathrm{a}}$ & $15.44 \pm 5.66(36.68)$ & $6.60-21.43(6)$ & 17.18 & 12.45 & 14.59 \\
\hline \multicolumn{6}{|l|}{ Peroxiredoxins } \\
\hline PRDX1 & $53.52 \pm 11.78(22.01)$ & $30.73-64.38(6)$ & 56.35 & 66.81 & 63.62 \\
\hline PRDX2 & $34.70 \pm 3.60(10.37)$ & $27.95-38.49(6)$ & 35.49 & 27.12 & 36.83 \\
\hline PRDX3 & $17.67 \pm 2.50(14.18)$ & $14.14-21.48(6)$ & 17.48 & 28.16 & 10.99 \\
\hline PRDX4 & $7.55 \pm 2.96(39.20)$ & $3.81-11.92(6)$ & 6.96 & 37.50 & 4.57 \\
\hline PRDX5 & $26.93 \pm 4.86(18.05)$ & $18.85-31.72(6)$ & 28.55 & 18.77 & 23.50 \\
\hline PRDX6 & $34.29 \pm 6.64(19.36)$ & $22.85-39.92(6)$ & 36.96 & 23.23 & 33.52 \\
\hline FAM213A & $16.63 \pm 7.25(43.62)$ & $8.04-24.01(5)$ & 18.57 & & 6.70 \\
\hline \multicolumn{6}{|c|}{ Methanethiol oxidase } \\
\hline SELENBP1 ${ }^{\mathrm{a}}$ & $15.45 \pm 2.16(13.98)$ & $12.52-17.52(5)$ & 15.61 & 6.67 & 12.30 \\
\hline \multicolumn{6}{|c|}{ Disulfide-isomerases } \\
\hline $\mathrm{P} 4 \mathrm{HB}$ & $26.09 \pm 6.99(26.77)$ & $19.16-37.98(6)$ & 24.39 & 51.53 & 14.86 \\
\hline PDIA3 & $21.82 \pm 8.73(40.01)$ & $11.87-37.20(6)$ & 20.28 & 40.86 & 12.07 \\
\hline PDIA4 & $7.17 \pm 1.43(19.89)$ & $4.98-8.66(6)$ & 7.62 & 9.31 & 4.35 \\
\hline PDIA6 & $22.34 \pm 11.09(49.63)$ & $11.67-42.56(6)$ & 19.33 & 46.98 & 12.22 \\
\hline \multicolumn{6}{|c|}{ Scavenger receptors } \\
\hline SSC5D & $5.30 \pm 2.61(49.26)$ & $2.26-8.82(5)$ & 5.00 & 4.16 & \\
\hline $\mathrm{CD} 163^{\mathrm{b}}$ & $9.16 \pm 3.71(40.52)$ & $4.61-12.83(5)$ & 10.57 & & 4.79 \\
\hline
\end{tabular}

CAT, catalase; GSS, glutathione synthetase; P4HB, prolyl 4-hydroxylase.

${ }^{a}$ According to Human Protein Atlas, these enzymes are reported to be present in the skin at the RNA level (low copy numbers) but not at the protein level.

${ }^{b}$ According to Human Protein Atlas, these enzymes are not reported to be present in the skin at the RNA or protein level.

Table 6. In human skin, the most abundant peptidase/protease was dermicidin $\left(30.98 \pm 20.58 \mathrm{pmol} \mathrm{mg}^{-1}\right.$ protein) and was expressed in similar abundance in LabSkin 2. This protein was found in low abundance in LabSkin 1. The second most abundant protein in human skin was cathepsin (CTS) D (24.35 $\pm 6.18 \mathrm{pmol} \mathrm{mg}^{-1}$ protein). This enzyme was also quantified in LabSkin at similar levels. The most abundant peptidase/protease inhibitors were cystatin (CST) B (42.93 \pm $22.3 \mathrm{pmol} \mathrm{mg}^{-1}$ protein), which was expressed at similar levels in both human skin and LabSkin, and plasma protease $\mathrm{C} 1$ inhibitor (SERPING1) $\left(24.68 \pm 6.61 \mathrm{pmol} \mathrm{mg}^{-1}\right.$ protein), which was much less abundant in LabSkin compared with the human skin.

Assessment of $\mathbf{P 4 5 0}$ and Esterase Activity in LabSkin by Substrate-Based MALDI Imaging. From the results of the labelfree quantitative proteomics, $\mathrm{P} 450$ enzymes were almost absent in human skin and LabSkin, with only CYP51A1, CYP7B1, and
CYP8A1 quantified in human skin at less than $5 \mathrm{pmol} \mathrm{mg}^{-1}$ protein. In contrast, enzymes with potential esterase activity, such as serum paraoxonase/arylesterase $1(\mathrm{PON} 1)\left(11.28 \pm 1.99 \mathrm{pmol} \mathrm{mg}^{-1}\right.$ total protein), CES1 (14.43 $\pm 1.91 \mathrm{pmol} \mathrm{mg}^{-1}$ total protein), and esterase $\mathrm{D}$ (ESD) $\left(15.15 \pm 3.72 \mathrm{pmol} \mathrm{mg}^{-1}\right.$ total protein), were quantified at abundance levels equivalent to a majority of the XME in human skin and LabSkin. MALDI-MSI experiments were performed to examine the P450 and esterase activity in LabSkin using the substrate-based MSI approach. To examine P450 activity, LabSkin tissue was treated with terbinafine, a substrate for CYP2C9, CYP1A2, and CYP3A4, and tolbutamide, a CYP2C9 substrate, prior to substrate-based MSI. No metabolite formation was observed, indicating the absence of CYP2C9, CYP1A2, and CYP3A4 activity in the LabSkin (Fig. 4). This is in agreement with the label-free quantitative proteomics data in which these enzymes were not identified or quantified. To examine esterase 
TABLE 6

Expression levels of proteases, nucleases, and protease inhibitors in human skin and LabSkin

Protein identification and quantification was based on the presence of at least two different peptides and from human skin of at least two donors. Protein expression is represented by the mean, the S.D. of the mean (S.D.), the percent CV, the range (min-max), and the number of samples (n). Protein abundance is reported in picomole milligram ${ }^{-1}$ of protein.

\begin{tabular}{|c|c|c|c|c|c|}
\hline \multirow{2}{*}{ Protein } & \multicolumn{3}{|c|}{ Human Skin } & \multirow{2}{*}{ LabSkin 1} & \multirow{2}{*}{ LabSkin 2} \\
\hline & Mean \pm S.D. $(\mathrm{CV})$ & Range $(n)$ & Median & & \\
\hline \multicolumn{6}{|c|}{ Aminopeptidases } \\
\hline ANPEP & $13.03 \pm 10.09(77.39)$ & $5.48-27.33(4)$ & 9.66 & 22.74 & \\
\hline DNPEP & $9.93 \pm 2.47(24.83)$ & $7.10-11.58(3)$ & 11.12 & & 1.51 \\
\hline ERAP1 & $4.63 \pm 1.09(23.55)$ & $2.91-5.88(6)(3)$ & 4.92 & 5.35 & 3.36 \\
\hline LAP3 & $10.14 \pm 4.56(44.94)$ & $4.59-16.93(6)$ & 9.77 & 6.95 & 5.36 \\
\hline LNPEP & $6.42 \pm 2.40(37.42)$ & $4.72-8.12(2)$ & 6.42 & & \\
\hline METAP1 & $3.10 \pm 0.66(21.19)$ & $2.63-3.56(2)$ & 3.10 & 9.24 & 1.68 \\
\hline NPEPPS $^{\mathrm{a}}$ & $7.99 \pm 1.51(18.92)$ & $5.91-9.61(6)$ & 8.05 & 9.51 & 5.76 \\
\hline RNPEP & $19.51 \pm 28.19(144.45)$ & $5.60-76.85(6)$ & 8.54 & 42.01 & 71.51 \\
\hline XPNPEP1 & $3.90 \pm 1.70(43.57)$ & $2.40-6.32(4)$ & 3.44 & 5.41 & \\
\hline XPNPEP3 & $8.34 \pm 0.50(5.99)$ & $7.77-8.67(3)$ & 8.58 & & 5.38 \\
\hline \multicolumn{6}{|l|}{ Endopeptidases } \\
\hline ASPRV1 & $11.12 \pm 2.94(26.47)$ & $6.18-14.54(6)$ & 11.26 & 30.14 & 7.58 \\
\hline CAPN1 & $12.54 \pm 2.14(17.04)$ & $10.63-15.72(6)$ & 11.85 & 12.61 & 9.86 \\
\hline CAPN2 & $9.99 \pm 2.57(25.73)$ & $5.94-12.85(5)$ & 10.42 & 5.45 & 8.25 \\
\hline FAP & $4.50 \pm 1.44(31.93)$ & $2.93-5.76(3)$ & 4.80 & 5.14 & \\
\hline PREP & $6.28 \pm 1.76(28.06)$ & $4.51-8.83(6)$ & 5.89 & 5.15 & 3.64 \\
\hline \multicolumn{6}{|c|}{ Peptidases/proteases } \\
\hline CNDP2 & $11.59 \pm 3.45(29.76)$ & $5.16-15.59(6)$ & 12.17 & 5.45 & 8.23 \\
\hline DPP3 & $4.94 \pm 1.88(38.15)$ & $2.98-8.06(5)$ & 4.59 & 5.69 & 3.61 \\
\hline $\mathrm{DPP}^{\mathrm{a}}$ & $4.80 \pm 2.00(41.63)$ & $2.99-7.65(4)$ & 4.28 & 6.17 & \\
\hline DPP7 & $2.81 \pm 1.78(63.27)$ & $1.55-4.06(2)$ & 2.81 & 3.30 & \\
\hline LONP1 & $4.51 \pm 0.99(21.85)$ & $3.40-5.29(3)$ & 4.85 & 5.70 & 1.95 \\
\hline PEPD & $6.22 \pm 1.85(29.75)$ & $3.78-8.71(6)$ & 6.45 & 3.81 & 5.21 \\
\hline TPP1 & $10.57 \pm 2.27(21.50)$ & $7.48-13.66(5)$ & 10.52 & 12.08 & 5.66 \\
\hline CTSA & $5.26 \pm 2.30(43.64)$ & $2.28-8.60(5)$ & 4.75 & 14.37 & 3.06 \\
\hline CTSB & $8.95 \pm 2.56(28.54)$ & $4.49-11.53(6)$ & 9.70 & 9.27 & 5.03 \\
\hline CTSC & $7.72 \pm 4.41(57.13)$ & $5.41-15.59$ & 5.84 & 20.44 & 2.73 \\
\hline CTSD & $24.35 \pm 6.18(25.38)$ & $17.65-32.76(6)$ & 23.59 & 19.12 & 11.39 \\
\hline $\mathrm{CTSG}^{\mathrm{b}}$ & $9.50 \pm 6.73(70.84)$ & $4.74-14.26(2)$ & 9.50 & & 2.51 \\
\hline CTSZ & $8.06 \pm 3.12(38.75)$ & $4.25-12.62(5)$ & 8.27 & 16.80 & 3.60 \\
\hline $\mathrm{DCD}^{\mathrm{c}}$ & $30.98 \pm 20.58(66.44)$ & $10.73-60.60(5)$ & 20.73 & 4.53 & 49.02 \\
\hline BLMH & $7.08 \pm 2.22(31.36)$ & $5.75-9.65(3)$ & 5.86 & & \\
\hline \multicolumn{6}{|l|}{ Metalloproteases } \\
\hline ADAM10 & $3.13 \pm 0.55(17.46)$ & $2.75-3.52(2)$ & 3.13 & & \\
\hline $\mathrm{MMP}^{\mathrm{a}}$ & $4.99 \pm 5.49(110.16)$ & $1.10-8.87(2)$ & 4.99 & 8.08 & \\
\hline \multicolumn{6}{|c|}{ Carboxypeptidases } \\
\hline $\mathrm{CPA} 3^{\mathrm{a}}$ & $9.56 \pm 4.67(48.82)$ & $4.59-15.00(4)$ & 9.33 & & \\
\hline $\mathrm{CPB} 2^{\mathrm{b}}$ & $5.63 \pm 1.79(31.83)$ & $2.91-7.90(5)$ & 5.92 & & 2.98 \\
\hline $\mathrm{CPM}^{\mathrm{a}}$ & $3.89 \pm 1.60(41.09)$ & $2.76-5.02(2)$ & 3.89 & & \\
\hline $\mathrm{CPN} 1^{\mathrm{b}}$ & $10.13 \pm 2.23(21.98)$ & $8.56-11.71(2)$ & 10.13 & & \\
\hline $\mathrm{CPN} 2^{\mathrm{b}}$ & $6.93 \pm 1.36(19.60)$ & $4.66-8.11(5)$ & 7.13 & & 2.45 \\
\hline $\mathrm{CPQ}^{\mathrm{a}}$ & $3.92 \pm 1.04(26.49)$ & $2.69-5.38(5)$ & 4.07 & 4.63 & 2.28 \\
\hline $\mathrm{CPVL}^{\mathrm{a}}$ & $2.85 \pm 0.92(32.30)$ & $1.98-3.82(3)$ & 2.75 & & \\
\hline PRCP & $4.79 \pm 2.27(47.45)$ & $2.97-9.20(6)$ & 4.13 & 10.76 & \\
\hline \multicolumn{6}{|l|}{ Nucleases } \\
\hline ENDOD1 & $4.57 \pm 2.66(58.22)$ & $2.18-7.48(4)$ & 4.30 & 4.08 & \\
\hline RNASE $^{\text {a }}$ & $9.46 \pm 1.79(18.87)$ & $7.54-11.64(5)$ & 8.80 & & \\
\hline RNASE4 $^{\mathrm{a}}$ & $3.78 \pm 1.98(52.39)$ & $1.41-6.22(4)$ & 3.74 & & 3.14 \\
\hline RNASET2 & $6.57 \pm 1.39(21.14)$ & $4.58-7.65(4)$ & 7.03 & 5.55 & \\
\hline SND1 & $3.43 \pm 2.35(68.57)$ & $1.38-7.44(5)$ & 2.79 & 9.25 & \\
\hline TATDN1 & $5.55 \pm 2.21(39.73)$ & $2.54-7.49(4)$ & 6.09 & & \\
\hline $\mathrm{YBX}^{\mathrm{d}}$ & $2.73 \pm 1.43(52.45)$ & $1.22-4.50(4)$ & 2.60 & 5.55 & \\
\hline \multicolumn{6}{|c|}{ Peptidase/protease inhibitors } \\
\hline CST3 & $9.88 \pm 3.61(36.54)$ & $6.07-14.71(5)$ & 9.58 & 18.39 & 6.21 \\
\hline CST6 & $8.66 \pm 3.01(34.80)$ & $5.58-12.82(5)$ & 8.42 & & \\
\hline CSTA & $9.24 \pm 5.47(59.24)$ & $5.37-13.10(2)$ & 9.24 & 15.59 & \\
\hline CSTB & $42.93 \pm 22.3(52.0)$ & $25.65-82.24(6)$ & 34.01 & 38.07 & 37.26 \\
\hline PI16 & $8.74 \pm 2.83(32.37)$ & $5.61-13.24(5)$ & 7.84 & & 4.24 \\
\hline SERPING $1^{\mathrm{a}}$ & $24.68 \pm 6.61(26.79)$ & $13.15-29.79(5)$ & 26.39 & 3.26 & 13.21 \\
\hline SPINK5 & $7.06 \pm 2.02(28.64)$ & $5.63-8.49(2)$ & 7.06 & 3.96 & \\
\hline Proteosome & & & & & \\
\hline PSMC1 & $6.89 \pm 1.04(15.02)$ & $6.16-7.62(2)$ & 6.89 & 1.82 & \\
\hline PSMC2 & $2.61 \pm 0.96(36.79)$ & $1.24-3.42(4)$ & 2.88 & 4.25 & 1.78 \\
\hline PSMC3 & $5.22 \pm 1.45(27.83)$ & $3.38-7.74(6)$ & 5.02 & 5.86 & 3.24 \\
\hline PSMC5 & $2.95 \pm 0.86(29.03)$ & $1.69-3.59(4)$ & 3.26 & 4.41 & \\
\hline PSMC6 & $3.80 \pm 1.09(28.76)$ & $2.82-5.53(5)$ & 3.54 & 6.66 & 1.99 \\
\hline Ribonuclease in & & & & & \\
\hline RNH1 & $22.47 \pm 5.55(24.71)$ & $12.60-25.88(5)$ & 24.34 & 21.65 & 17.56 \\
\hline
\end{tabular}

DCD, dermicidin; SERPING1, plasma protease C1 inhibitor 
${ }^{a}$ According to Human Protein Atlas, these enzymes are reported to be present in the skin at the RNA level (low copy numbers) but not at the protein level ${ }^{b}$ According to Human Protein Atlas, these enzymes are not reported to be present in the skin at the RNA or protein level.

${ }^{c}$ Also displays antimicrobial activity, thereby limiting skin infection by potential pathogens in the first few hours after bacterial colonization.

${ }^{d}$ YBX1 can promote the expression of multidrug resistance 1 gene, which is involved in the development of drug resistance.

activity, LabSkin was treated with $0.5 \%(\mathrm{w} / \mathrm{w})$ of human carboxylesterase 1 (hCES1) substrate, methylphenidate hydrochloride, for 24 hours. Figure 5 shows MALDI-MSI images of the distribution of methylphenidate ion at $m / z 234$ and the metabolite, ritalinic acid ion at $\mathrm{m} / \mathrm{z}, 220$ in both blank and treated LabSkin sections recorded at $60 \mu \mathrm{m}$ spatial resolution. It can be seen that the metabolite ritalinic acid signal appeared to be localized in the outer layer of skin, that is, the epidermis.

\section{Discussion}

In Europe, although animal testing of cosmetic ingredients has been discontinued, topical drugs are still tested on animals, and there is clearly a need for alternative model systems to assess the safety and efficacy of both topical drugs and cosmetic ingredients. Although several artificial human skin models have been proposed, they are limited by a lack of understanding of xenobiotic metabolism and transport in human skin. Efforts have been made to understand XME gene expression in human skin and in vitro skin models using reverse transcription polymerase chain reaction (Kazem et al., 2019); however, it is increasingly accepted that mRNA abundance is a poor proxy for protein abundance and activity (Maier et al., 2009; Couto et al., 2020). In this study, we used a combination of label-free quantitative mass spectrometry and substrate-based mass spectrometry imaging to assess xenobiotic metabolism in human skin and an in vitro artificial skin model (LabSkin) and have quantified several XMEs for the first time in these systems.
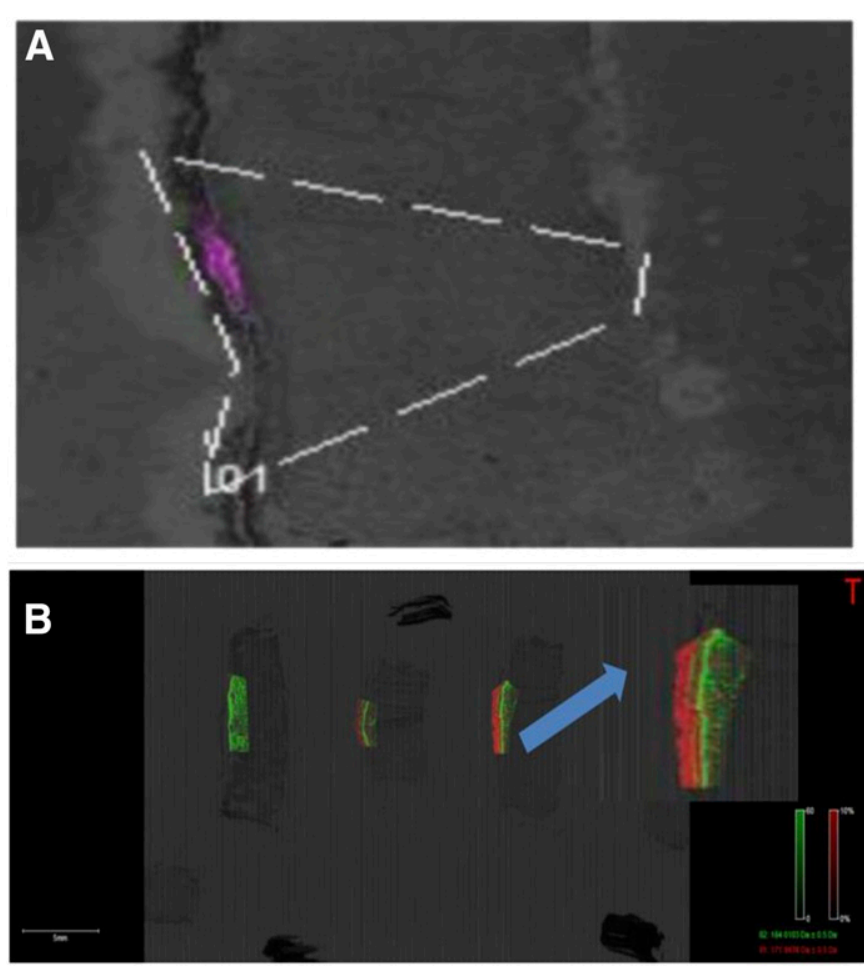

Fig. 4. MALDI-MSI on a LabSkin section treated with terbinafine (A) and tolbutamide (B). Terbinafine is a substrate for CYP2C9, CYP1A2, and CYP3A4. (A) No evidence of metabolite formation was observed in terbinafine-treated LabSkin section (purple). Tolbutamide is a substrate for CYP2C9. The tolbutamide is shown in red, and phosphatidylcholine lipid head group is shown in green. (B) No evidence of metabolite formation was observed. Arrow indicates a magnified image.
Xenobiotic metabolism is divided into three phases: oxidation (phase I), addition of polar groups to phase I metabolites (phase II), and clearance from cells (phase III). A single, pioneering mass spectrometry-based study on xenobiotic metabolism in skin focused on phase I and phase II enzymes only (van Eijl et al., 2012). We have now quantified phase I and phase II enzymes and also transporters and accessory enzymes involved in xenobiotic metabolism using mass spectrometry.

In the liver, phase I metabolism is dominated by enzymes from the P450 family (Couto et al., 2019), but there is disagreement about the importance of P450 enzymes in skin (van Eijl et al., 2012; Kazem et al., 2019). We have identified three P450 enzymes, CYP7B1, CYP8A1, and CYP51A1, primarily involved in endogenous metabolism. CYP7B1 and CYP51A1 are involved in cholesterol metabolism; CYP8A1 is involved in eicosanoids metabolism (Lepesheva and Waterman, 2007; Li et al., 2008; Yantsevich et al., 2014). P450 enzymes with a known role in xenobiotic metabolism, such as CYP1A2, CYP2C9, and CYP3A4, were neither identified nor quantified. Substrate-based mass spectrometry imaging confirmed the absence of the activity of these P450 enzymes in LabSkin. This observation agrees with previous studies by mass spectrometry-based proteomics and Western blots where the enzymes from P450 families 1, 2, 3, and 4 were not detected (van Eijl et al., 2012). Conventionally, $\mathrm{P} 450$ activity in human skin has been assessed by either 7-ethoxy resorufin O-deethylase assay for CYP1 enzymes, 7-methoxy4-(trifluoromethyl)-coumarin O-demethylase assay for CYP2 enzymes, or the benzyloxy-4-trifluoromethylcoumarin-O-debenzy-loxylase assay

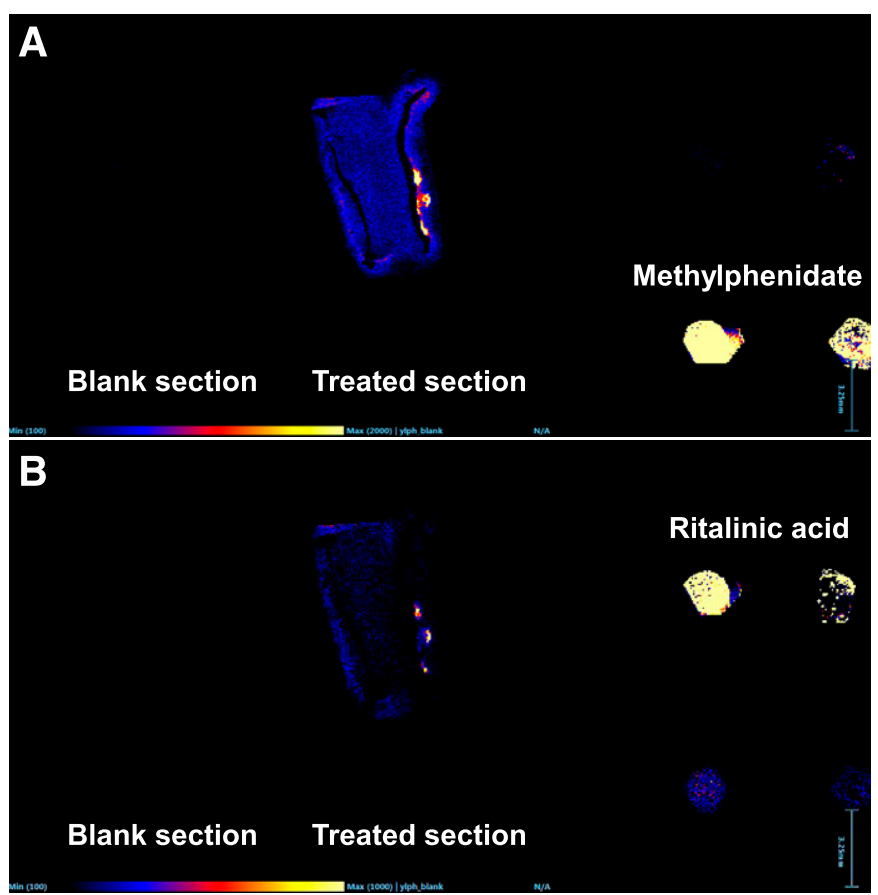

Fig. 5. MALDI-MSI on a LabSkin section treated with methylphenidate $(0.5 \% \mathrm{w} / \mathrm{w})$ for 24 hours showing the distribution of methylphenidate peak at $\mathrm{m} / \mathrm{z}$ 234(A) and ritalinic acid peak at $m / z 220$ (B). Untreated LabSkin (blank) sections were included as controls (left) alongside methylphenidate-treated LabSkin section (middle). Standard ritalinic acid (top) and methylphenidate (bottom) were spotted at a concentration of $1 \mathrm{mg} \mathrm{ml}^{-1}$ alongside the LabSkin sections as can be seen toward the left of the images. Mass spectrometry images clearly show presence of the substrate, methylphenidate, in the epidermis (A) and the presence of the product of esterase activity, ritalinic acid, in the epidermis (B). 
for CYP3 enzymes. These studies reported activity either close to the limit of detection or below the limit of detection (Kazem et al., 2019). mRNA transcripts of P450 enzymes have, however, been quantified in native human skin, suggesting that $\mathrm{P} 450$ expression may be regulated at the level of protein translation. It has been suggested that certain P450 enzymes are induced in the presence of specific substrates (Ahmad and Mukhtar, 2004). By contrast with human skin, P450 activity has been established conclusively in the skin of mouse models (Oesch et al., 2014), indicating that animal models may have limited applicability to human products. With regard to the LabSkin assessed in this study, the protein abundance of P450 and auxiliary proteins in LabSkin 1 were comparable to ex vivo human skin, whereas no P450 enzymes were detected in LabSkin 2.

Other phase I xenobiotic metabolising enzymes-dehydrogenases, reductases, esterases, and hydrolases-were quantified in our study. Although the abundance of these proteins has not been previously measured, the presence of these proteins in native human skin has been previously reported (van Eijl et al., 2012). Carboxylesterase activity has not been previously demonstrated in human skin in spite of the quantification of the cognate mRNA in HaCaT keratinocytes and skin models (Kazem et al., 2019). We quantified CES1 and CES1P1 in human skin. Although, the abundance of these enzymes was lower in LabSkin, substrate-based mass spectrometry imaging was used to evaluate CES1 activity for the first time, and indeed, CES1 activity was found in LabSkin.

The important role of protein glutathionylation in skin is highlighted by the identification and quantification of several GSTs isoforms among phase II xenobiotic metabolizing enzymes. GST activity in human skin has been confirmed in previous studies using a broad spectrum GST substrate as well as through gene expression and proteomic studies (van Eijl et al., 2012; Kazem et al., 2019). In agreement with previous studies, GST pi was the most abundant GST enzyme in our study. Reversible protein glutathionylation is known to play a role in cellular regulation, signaling transduction, and antioxidant defense (Dalle-Donne et al., 2007; Gallogly and Mieyal, 2007). Moreover, glutathione conjugates may be further processed and secreted, and further metabolism occurs extracellularly (Couto et al., 2016). We also quantified sulfotransferase enzymes in the human skin explants, which were not detected previously (van Eijl et al., 2012). Although SULTs are absent in mouse skin, they are essential for the metabolism of endogenous compounds in humans (Oesch et al., 2014; Kazem et al., 2019). The abundance of GST isoforms and other transferase enzymes in LabSkin was similar to native human skin. SULT enzymes were not detected in LabSkin. The abundance of SULT mRNA has been observed to increase upon calcium-induced differentiation of human keratinocytes (Oesch et al., 2018). Therefore, our inability to detect SULTs in the LabSkin models may be linked to the in vitro culturing conditions. Evidence for the presence of mRNA of SULT enzymes and SULT activity in human skin has been reported (Kazem et al., 2019). We were also able to confidently quantify COMT for the first time.

Although mRNA of UGT and N-acetyltransferase enzymes have been quantified in low abundance in human skin, our results agree with previous proteomics studies where these enzymes were not detected in human skin (van Eijl et al., 2012). Conflicting observations between activity assays, mRNA abundance, and proteomics studies may be because these enzymes are preferentially expressed in certain human anatomic sites or because the expression of these proteins are controlled at the level of protein translation. More studies are needed to shed light on the role of regulatory cellular processes in human skin.

The use of unbiased, label-free, quantitative proteomics approach to lysates of epidermal cells and LabSkin provided us with an opportunity to detect and quantify transporter proteins. Although the use of membrane-based protein preparations would enable a comprehensive insight into the transporters in the human skin epidermis, using our total cellular protein extract, we were able to confidently quantify two ABC and eight SLC transporters, presumably those of highest abundance. The mRNA abundance of certain ABC and SLC transporters has been quantified in human keratinocytes and ex vivo human skin (Fujiwara et al., 2014; Osman-Ponchet et al., 2014). The mRNA transcripts of ABCC3 has been established to be the highest is human skin, although large interindividual variability is reported among the Caucasian population (Takechi et al., 2018). We did not detect ABCC3 in our study but found good evidence of ABCA8 and ABCB11. Similarly, the mRNA transcripts of SLC22A3 and SLCO3A1 have been established to be the highest in human skin (Takechi et al., 2018), but we detected neither SLC22A3 nor SLCO3A1 at the protein level. ABCA8 is known to be involved in ATP-dependent lipophilic drug transport (Tsuruoka et al., 2002), and $A B C B 11$ is involved in the secretion of conjugated bile salts (Hayashi et al., 2005). The SLC transporter proteins detected in the human skin in our study are associated with endogenous metabolism, and some play important roles in skin conditions (Nakamura et al., 2010; Gomez et al., 2013). SLC25A5 is known to be highly expressed in psoriasis compared with normal skin (Lundberg et al., 2015), and SLC12A2 may be involved in transport of fumaric acid esters used to treat psoriasis (Onderdijk et al., 2014). SLC12A2 has been quantified for the first time in this study. In LabSkin, apart from SLC25A6 and SLC44A1, the abundance of transporters was low and in some cases undetected.

The label-free approach also allowed us to quantify antioxidant enzymes in human skin and LabSkin. Antioxidant enzymes play an important auxiliary role in xenobiotic metabolism, as they sequester the reactive oxygen species produced during phase I xenobiotic metabolism (Couto et al., 2016) but are not routinely investigated when studying xenobiotic metabolism. To our knowledge, the abundance of antioxidant enzymes in skin, liver, and kidney has not been reported to date. We contend that incorporation of the analysis of antioxidants and antioxidant enzymes when studying xenobiotic metabolism is important. Xenobiotic metabolism has the potential to upset the fine redox balance maintained in human skin and lead to skin conditions such as psoriasis, atopic dermatitis, and cancer (BenYehuda Greenwald et al., 2016; Couto et al., 2016). Our results indicate that the human skin contains a high diversity and abundance of redox enzymes, which can be mimicked in LabSkin.

We hypothesize that proteases and peptidases also play an important role in maintaining cellular homeostasis in skin exposed to xenobiotics by regulating phase I enzymes, phase II enzymes, transporters, and redox enzymes. Indeed, dysregulation of proteases and peptidases in human skin is known to affect skin hydration and maintenance of skin integrity (Verdier-Sévrain and Bonté, 2007). Moreover, bioactive peptides and proteins are increasingly employed in topical drugs and cosmetics, and the presence and activity of proteases and peptidases in skin can alter the lifespan of these additives in topical drugs and cosmetics. To our knowledge, this is the first report of the abundance of proteases and peptidases in human skin, and the most abundant proteases and peptidases in human skin were also detected in LabSkin.

Although a wide variety of XMEs, transporters and auxiliary proteins were quantified in human skin, high interindividual variability was seen in the presence and abundance of these proteins across the six samples used in this study (columns 2 and 3 in Tables 2-6). The modest number $(n=6)$ of human skin samples studied here precludes a deeper analysis of the reasons underlying the high 
interindividual variability. Such high interindividual variability in $\mathrm{XME}$ and transporter abundance has also been reported in mass spectrometry-based quantitative proteomics studies of human liver and intestine (Couto et al., 2019, 2020). A similarly high interbatch variability is seen across the two independent replicates of LabSkin. Despite this, predominantly, proteins that were present in all six human skin samples were also found in both replicates of LabSkin. Interbatch differences observed in LabSkin may be due to differences in the level of differentiation of keratinocytes at the point of analysis. For example, LabSkin 2 lacked detectable stratum corneum markers, suggesting that it may have had a more immature phenotype than LabSkin 1. However, a larger study involving a greater number of LabSkin batches and a greater number of human skin samples will allow a better understanding of the sources of interbatch and interindividual variability.

In conclusion, we report for the first time the protein abundance of XMEs, transporters, and auxiliary enzymes involved in xenobiotic metabolism in human skin and LabSkin. Improved understanding of skin physiology will aid validation of in vitro living skin equivalents and the development of robust mathematical models, including physiologically based pharmacokinetic tools, which can be used to predict drug metabolism and disposition in human skin, with applications such as predicting bioavailability of topical preparations. Knowledge of the abundance of drug metabolizing enzymes, transporters, and drug targets in human skin as well as perturbations of expression due to skin conditions, such as psoriasis, can help predict changes in patients' response to therapy, leading to better tailored dosage regimens. The in vitro living skin equivalent model, LabSkin, evaluated in this study was shown to express a number of proteins present in human skin. Although the abundance of some of these proteins is equivalent to skin, a few proteins either are not detected or have low abundance. The data in our study can guide the refinement of culturing conditions to ensure that the proteomic profile of LabSkin is representative of in vivo human skin to confidently reduce, refine, and replace the use of animals in development of safe and efficacious topical drugs and cosmetics. In particular, we anticipate that the inclusion of the abundance of redox enzymes and proteases and peptidases in mathematical models will enable such models to become more robust and representative of xenobiotic metabolism in human skin.

\section{Acknowledgments}

The authors thank Eleanor Savill for helping to format and submit the manuscript.

\section{Authorship Contributions}

Participated in research design: Couto, Newton, Sidaway, RostamiHodjegan, Clench, Barber.

Conducted experiments: Couto, Newton, Russo, Karunakaran, Al-Majdoub. Contributed new reagents or analytical tools: Couto, Russo.

Performed data analysis: Couto, Newton, Russo, Karunakaran, Achour.

Wrote or contributed to the writing of the manuscript: Couto, Karunakaran, Newton, Russo, Achour, Sidaway, Rostami-Hodjegan, Clench, Barber.

\section{References}

Ahmad N and Mukhtar H (2004) Cytochrome p450: a target for drug development for skin diseases. J Invest Dermatol 123:417-425.

Al Feteisi H, Al-Majdoub ZM, Achour B, Couto N, Rostami-Hodjegan A, and Barber J (2018) Identification and quantification of blood-brain barrier transporters in isolated rat brain microvessels. J Neurochem 146:670-685.

Ben-Yehuda Greenwald M, Ben-Sasson S, Bianco-Peled H, and Kohen R (2016) Skin redox balance maintenance: the need for an Nrf2-activator delivery system. Cosmetics 3:1.

Bradford MM (1976) A rapid and sensitive method for the quantitation of microgram quantities of protein utilizing the principle of protein-dye binding. Anal Biochem 72:248-254.
Cantòn I, Cole DM, Kemp EH, Watson PF, Chunthapong J, Ryan AJ, MacNeil S, and Haycock JW (2010) Development of a 3D human in vitro skin co-culture model for detecting irritants in realtime. Biotechnol Bioeng 106:794-803.

Chau DYS, Johnson C, MacNeil S, Haycock JW, and Ghaemmaghami AM (2013) The development of a 3D immunocompetent model of human skin. Biofabrication 5:035011.

Couto N, Al-Majdoub ZM, Achour B, Wright PC, Rostami-Hodjegan A, and Barber J (2019) Quantification of proteins involved in drug metabolism and disposition in the human liver using label-free global proteomics. Mol Pharm 16:632-647.

Couto N, Al-Majdoub ZM, Gibson S, Davies PJ, Achour B, Harwood MD, Carlson G, Barber J, Rostami-Hodjegan A, and Warhurst G (2020) Quantitative proteomics of clinically relevant drug-metabolizing enzymes and drug transporters and their intercorrelations in the human small intestine. Drug Metab Dispos 48:245-254.

Couto N, Malys N, Gaskell SJ, and Barber J (2013) Partition and turnover of glutathione reductase from Saccharomyces cerevisiae: a proteomic approach. J Proteome Res 12:2885-2894.

Couto N, Wood J, and Barber J (2016) The role of glutathione reductase and related enzymes on cellular redox homoeostasis network. Free Radic Biol Med 95:27-42.

Craik CS, Page MJ, and Madison EL (2011) Proteases as therapeutics. Biochem J 435:1-16.

Dalle-Donne I, Rossi R, Giustarini D, Colombo R, and Milzani A (2007) S-glutathionylation in protein redox regulation. Free Radic Biol Med 43:883-898.

El-Khateeb E, Vasilogianni A-M, Alrubia S, Al-Majdoub ZM, Couto N, Howard M, Barber J, Rostami-Hodjegan A, and Achour B (2019) Quantitative mass spectrometry-based proteomics in the era of model-informed drug development: applications in translational pharmacology and recommendations for best practice. Pharmacol Ther 203:107397.

Flores C, Santos M, Pereira SB, Mota R, Rossi F, De Philippis R, Couto N, Karunakaran E, Wright PC, Oliveira P, et al. (2019) The alternative sigma factor SigF is a key player in the control of secretion mechanisms in Synechocystis sp. PCC 6803. Environ Microbiol 21:343-359.

Fujiwara R, Takenaka S, Hashimoto M, Narawa T, and Itoh T (2014) Expression of human solute carrier family transporters in skin: possible contributor to drug-induced skin disorders. Sci Rep 4:5251.

Gallogly MM and Mieyal JJ (2007) Mechanisms of reversible protein glutathionylation in redox signaling and oxidative stress. Curr Opin Pharmacol 7:381-391.

Gomez G, Nardone V, Lotfi-Emran S, Zhao W, and Schwartz LB (2013) Intracellular adenosine inhibits IgE-dependent degranulation of human skin mast cells. J Clin Immunol 33:1349-1359.

Hayashi H, Takada T, Suzuki H, Onuki R, Hofmann AF, and Sugiyama Y (2005) Transport by vesicles of glycine- and taurine-conjugated bile salts and taurolithocholate 3-sulfate: a comparison of human BSEP with rat Bsep. Biochim Biophys Acta 1738:54-62.

Hu J, Van den Steen PE, Sang Q-XA, and Opdenakker G (2007) Matrix metalloproteinase inhibitors as therapy for inflammatory and vascular diseases. Nat Rev Drug Discov 6:480-498.

Kazem S, Linssen EC, and Gibbs S (2019) Skin metabolism phase I and phase II enzymes in native and reconstructed human skin: a short review. Drug Discov Today 24: $1899-1910$.

Lepesheva GI and Waterman MR (2007) Sterol 14 $\alpha$-demethylase cytochrome P450 (CYP51), a P450 in all biological kingdoms. Biochim Biophys Acta 1770:467-477.

Li Y-C, Chiang C-W, Yeh H-C, Hsu P-Y, Whitby FG, Wang L-H, and Chan N-L (2008) Structures of prostacyclin synthase and its complexes with substrate analog and inhibitor reveal a ligandspecific heme conformation change. J Biol Chem 283:2917-2926.

Lundberg KC, Fritz Y, Johnston A, Foster AM, Baliwag J, Gudjonsson JE, Schlatzer D, Gokulrangan G, McCormick TS, Chance MR, et al. (2015) Proteomics of skin proteins in psoriasis: from discovery and verification in a mouse model to confirmation in humans. Mol Cell Proteomics 14:109-119.

Maier T, Güell M, and Serrano L (2009) Correlation of mRNA and protein in complex biological samples. FEBS Lett 583:3966-3973.

Mathes SH, Ruffner H, and Graf-Hausner U (2014) The use of skin models in drug development. Adv Drug Deliv Rev 69-70:81-102.

Nakamura T, Fujiwara R, Ishiguro N, Oyabu M, Nakanishi T, Shirasaka Y, Maeda T, and Tamai (2010) Involvement of choline transporter-like proteins, CTL1 and CTL2, in glucocorticoidinduced acceleration of phosphatidylcholine synthesis via increased choline uptake. Biol Pharm Bull 33:691-696.

Oesch F, Fabian E, Guth K, and Landsiedel R (2014) Xenobiotic-metabolizing enzymes in the skin of rat, mouse, pig, Guinea pig, man, and in human skin models. Arch Toxicol 88: $2135-2190$.

Oesch F, Fabian E, and Landsiedel R (2018) Xenobiotica-metabolizing enzymes in the skin of rat, mouse, pig, Guinea pig, man, and in human skin models. Arch Toxicol 92:2411-2456.

Onderdijk AJ, Balak DMW, Baerveldt EM, Florencia EF, Kant M, Laman JD, van IJcken WF, Racz E, de Ridder D, Thio HB, et al. (2014) Regulated genes in psoriatic skin during treatment with fumaric acid esters. Br J Dermatol 171:732-741.

Osman-Ponchet H, Boulai A, Kouidhi M, Sevin K, Alriquet M, Gaborit A, Bertino B, Comby P, and Ruty B (2014) Characterization of ABC transporters in human skin. Drug Metabol Drug Interact 29:91-100.

Overall CM and Dean RA (2006) Degradomics: systems biology of the protease web. Pleiotropic roles of MMPs in cancer. Cancer Metastasis Rev 25:69-75.

Parker JL, Lowry RC, Couto NAS, Wright PC, Stafford GP, and Shaw JG (2014) Maf-dependent bacterial flagellin glycosylation occurs before chaperone binding and flagellar T3SS export. Mol Microbiol 92:258-272.

Puente XS, Sánchez LM, Gutiérrez-Fernández A, Velasco G, and López-Otín C (2005) A genomic view of the complexity of mammalian proteolytic systems. Biochem Soc Trans 33:331-334.

Raut MP, Couto N, Karunakaran E, Biggs CA, and Wright PC (2019) Deciphering the unique cellulose degradation mechanism of the ruminal bacterium Fibrobacter succinogenes S85. Sci Rep 9:16542.

Russo C, Brickelbank N, Duckett C, Mellor S, Rumbelow S, and Clench MR (2018) Quantitative investigation of terbinafine hydrochloride absorption into a living skin equivalent model by MALDI-MSI. Anal Chem 90:10031-10038.

Russo DA, Couto N, Beckerman AP, and Pandhal J (2016) A metaproteomic analysis of the response of a freshwater microbial community under nutrient enrichment. Front Microbiol 7: 1172

Sengupta A, Lichti UF, Carlson BA, Ryscavage AO, Gladyshev VN, Yuspa SH, and Hatfield DL (2010) Selenoproteins are essential for proper keratinocyte function and skin development. PLoS One 5:e12249.

Silva JC, Gorenstein MV, Li G-Z, Vissers JPC, and Geromanos SJ (2006) Absolute quantification of proteins by LCMSE: a virtue of parallel MS acquisition. Mol Cell Proteomics 5:144-156. 
Takechi T, Hirota T, Sakai T, Maeda N, Kobayashi D, and Ieiri I (2018) Interindividual differences in the expression of ATP-binding cassette and solute carrier family transporters in human skin DNA methylation regulates transcriptional activity of the human ABCC3 gene. Drug Metab Dispos 46:628-635.

Tsuruoka S, Ishibashi K, Yamamoto H, Wakaumi M, Suzuki M, Schwartz GJ, Imai M, and Fujimura A (2002) Functional analysis of ABCA8, a new drug transporter. Biochem Biophys Res Commun 298:41-45.

Uhlen M, Fagerberg L, Hallstrom BM, Lindskog C, Oksvold P, Mardinoglu A, Sivertsson A, Kampf C, Sjostedt E, Asplund A, et al. (2015) Tissue-based map of the human proteome. Science 347:1260419.

van Eijl S, Zhu Z, Cupitt J, Gierula M, Götz C, Fritsche E, and Edwards RJ (2012) Elucidation of xenobiotic metabolism pathways in human skin and human skin models by proteomic profiling. PLoS One 7:e41721.

Verdier-Sévrain S and Bonté F (2007) Skin hydration: a review on its molecular mechanisms. $J$ Cosmet Dermatol 6:75-82.
Yantsevich AV, Dichenko YV, Mackenzie F, Mukha DV, Baranovsky AV, Gilep AA, Usanov SA, and Strushkevich NV (2014) Human steroid and oxysterol 7 $\alpha$-hydroxylase CYP7B1: substrate specificity, azole binding and misfolding of clinically relevant mutants. FEBS J 281:1700-1713 Zeeuwen PLJM (2004) Epidermal differentiation: the role of proteases and their inhibitors. Eur J Cell Biol 83:761-773.

Address correspondence to: Narciso Couto, Department of Chemical and Biological Engineering, University of Sheffield, Sir Robert Hadfield Building, Mappin Street, Sheffield S1 3JD, UK. E-mail: n.couto@sheffield.ac.uk; or Jill Barber, Centre for Applied Pharmacokinetic Research, University of Manchester, Stopford Building, Oxford Road, Manchester M13 9PT, UK. E-mail: jill.barber@manchester.ac.uk 